\title{
RMetS
}

Royal Meteorological Society

\section{An investigation of rotational influences on tropical-cyclone size and intensity}

\author{
Roger K. Smith, ${ }^{\mathrm{a} *}$ Christoph W. Schmidt ${ }^{\mathrm{a}}$ and Michael T. Montgomery ${ }^{\mathrm{b}}$ \\ ${ }^{a}$ Meteorological Institute, University of Munich, Germany \\ ${ }^{\mathrm{b}}$ Dept. of Meteorology, Naval Postgraduate School, Monterey, California and Hurricane Research Division, NOAA, USA \\ ${ }^{*}$ Correspondence to: R. K. Smith, Meteorological Institute, University of Munich, Theresienstrasse 37, 80333 Munich, \\ Germany. E-mail: roger.smith@lmu.de \\ ${ }^{\dagger}$ The contribution of this author was prepared as part of his official duties as a US Federal Government employee.
}

We investigate the rotational constraint on the intensity and size of a tropical cyclones using a minimal, three-layer, axisymmetric tropical-cyclone model. In the first of two sets of experiments, the same initial baroclinic vortex is spun up in a quiescent environment with different levels of background rotation, characterized by the Coriolis parameter, $f$. It is found that the strongest vortices, as characterized by their final intensity, develop in environments with intermediate background rotation. It is found also that there exists a similar optimum background rotation strength to obtain the largest storm as measured by the radius of gale-force winds. These results appear to be in line with those of classical laboratory experiments by Turner and Lilly, an analogy that we explore in the present article. While the analogy is found to have certain limitations, including the fact that spin-up of the maximum tangential winds in the inner-core in the model takes place in the boundary layer, the study raises aspects of tropical-cyclone dynamics that we believe to be of fundamental importance and require further investigation.

As an aid to understanding the foregoing results, a second set of calculations is carried out with the vortex forced by a prescribed radial profile of diabatic heating rate typical of that in the first set and with other moist processes excluded. For this distribution of heating rate, there is no optimum background rotation rate for intensity within a realistic range of values for $f$, implying that the relationship between the forcing strength and rotation strength is an important additional constraint in tropical cyclones. However, in these experiments, there is an optimum latitude for size, comparable with that in the first set of experiments. An interpretation is offered for these findings. Copyright (C) 2011 Royal Meteorological Society

Key Words: hurricane; typhoon; boundary layer; ambient rotation rate

Received 29 July 2010; Revised 10 April 2011; Accepted 16 May 2011; Published online in Wiley Online Library 1 August 2011

Citation: Smith RK, Schmidt CW, Montgomery MT. 2011. An investigation of rotational influences on tropical-cyclone size and intensity. Q. J. R. Meteorol. Soc. 137: 1841-1855. DOI:10.1002/qj.862

\section{Introduction}

*There is observational evidence to suggest that there is little relationship between the intensity of a tropical cyclone

*This paper is dedicated to the memory of our dear friend and colleague, Dr. Wolfgang Ulrich, whose untimely death in 2005 was a major setback and its size, measured, for example, by the maximum near-surface tangential component of wind speed and the radius of gale-force winds, respectively (e.g. Merrill, 1984;

to investigations on the present topic. Some of his early calculations set the scene for our subsequent recognition of the important dynamical role of the boundary layer in the spin-up of the tropical-cyclone core. 
Weatherford and Gray, 1988). Since these observations were reported, they have remained a puzzle and it has not been until recently that an explanation has been offered to account for them (see below). Much theoretical work has been focused on estimating the maximum possible intensity that a storm can achieve in a particular environment (Emanuel, 1986, 1988, 1995; Holland, 1997; Bister and Emanuel, $1998)^{\dagger}$. A critique of these theories and list of references is given by Camp and Montgomery (2001), who concluded that Emanuel's theory comes closest to providing a useful calculation of maximum intensity. However they noted also several shortcomings in the theory, arguing for the need for more basic research on the axisymmetric and asymmetric dynamics of hurricanes.

As far as we are aware, there are no theories to account for the range of sizes of tropical cyclones that are observed in nature. More fundamentally, there is a lack of understanding of what governs the size of a storm through its life cycle. Some early numerical simulations by Yamasaki (1968) showed that storms tend to be larger at higher latitudes, consistent with observations by Merrill (1984) and with later calculations by DeMaria and Pickle (1988). The latter authors showed, using an idealized, axisymmetric, three-layer model, that low-latitude storms are smaller than high-latitude storms, but low-latitude storms intensify more rapidly initially. In addition, they showed that the final intensity of storms does not vary appreciably (they say rapidly) with latitude. These authors suggested that 'the effect of latitude appears to be related to the radial positioning of the diabatic heating', and argued that 'the boundary-layer convergence and thus the diabatic heating occur much closer to the storm centre as the latitude is decreased'. They go on to speculate how the foregoing result might be generalized in a three-dimensional simulation, noting that if the absolute vorticity of the synoptic-scale flow had an effect similar to latitude, the initial formation of tropical cyclones might be favoured in regions where the large-scale vorticity of the surrounding flow is small'. They speculated also that the absolute vorticity of the synoptic-scale flow may be a factor that influences the size of tropical cyclones.

\subsection{Mechanisms for tropical-cyclone spin-up}

While the question of what constraints determine a tropical cyclone's size (measured, e.g. by the radius of galeforce winds) seems largely unanswered, recent progress has been made in understanding the reasons for the apparent lack of a strong relationship between their intensity and size (Smith et al., 2009). Building on recent studies of tropical-cyclone intensification in an idealized threedimensional numerical model by Nguyen et al. (2008) and Shin and Smith (2008), these authors showed that, from an axisymmetric perspective, there are two mechanisms ${ }^{\ddagger}$ for

\footnotetext{
${ }^{\dagger}$ In theoretical studies, the maximum tangential wind is usually the preferred intensity metric. In operational communities, however, intensity is defined as a sustained horizontal wind speed at the surface or anemometer level (e.g. $10 \mathrm{~m}$ ) over some time interval, either a $1 \mathrm{~min}$ or $10 \mathrm{~min}$ average. To keep with the basic research theme of this paper, we will adhere to the former definition unless noted otherwise.

¥The statement in Smith etal. (2009) that these mechanisms are independent is too strong; there must be a degree of coupling between them through boundary-layer dynamics, as discussed by Montgomery and Smith (2011).
}

vortex intensification in this framework, both involving the radial convergence of absolute angular momentum.

- The first mechanism is associated with the radial convergence of absolute angular momentum above the boundary layer in conjunction with its conservation. The convergence is produced by a system-scale radial gradient of diabatic heating rate associated with deep, inner-core convection in the presence of enhanced surface moisture fluxes. This mechanism has been articulated previously by many authors (e.g. Willoughby, 1979; Schubert and Hack, 1983). It explains why the vortex expands in size and may be interpreted in terms of balance dynamics.

- The second mechanism is associated with the radial convergence of absolute angular momentum within the boundary layer and becomes progressively important in the inner core. Although absolute angular momentum is not materially conserved in the boundary layer, large wind speeds can be achieved there if the radial inflow is sufficiently large to bring the air parcels to small radii with minimal loss of angular momentum. This mechanism is tied fundamentally to the dynamics of the boundary layer, where the flow is not in gradient wind balance and deviations therefrom are significant over a substantial radial stretch.

The existence of these two mechanisms provides a plausible physical explanation for the long-standing observations of typhoons by Weatherford and Gray (1988), which indicate that inner-core changes in the azimuthalmean tangential wind speed often occur independently from those in the outer core. Given these new insights, it is natural to investigate to what extent the intensity and size are constrained by the level of ambient rotation, characterized here by the background vertical rotation rate.

The existence of the two spin-up mechanisms raises questions also concerning Emanuel's steady-state tropicalcyclone model (Emanuel, 1986) and his time-dependent models (Emanuel, 1989, 1995, 1997, 2004). These models focus largely on thermodynamic processes, making drastic simplifications to the dynamics through the assumption of gradient wind balance and hydrostatic balance. It was only recently that the tacit assumption of gradient wind balance in the boundary layer and its implications were noticed (Smith et al., 2008). The azimuthal momentum equation is used explicitly only in the boundary layer and only then to determine the radial (sic) inflow there. The assumption of strict gradient wind balance in the boundary layer, or even quasi-balanced dynamics associated with a generalized Ekman layer, is not supported by a scale analysis of the equations of motion (Smith and Montgomery, 2008; Vogl and Smith, 2009). The unbalanced dynamics in the innercore region are generally important for determining the maximum radial and tangential wind components that can be attained, and therefore important in determining the azimuthal-mean intensity of the vortex (Smith et al., 2008, 2009). The strong inflow that is a prominent feature of the inner-core boundary layer owes much of its magnitude to gradient wind imbalance (Bui et al., 2009). The lack of a clear explanation ${ }^{\S}$ given by Emanuel (1997) to the dynamical

${ }^{\S}$ Recently, Emanuel (2010, personnal communication) has provided some clarification of this issue, noting that there is inflow above the 
mechanism of spin-up obscures our ability to understand the potential dependence of his time-dependent theory on the ambient rotation.

A possible conceptual framework for investigating some of the issues raised above is provided by some early studies of geophysical vortices, which are discussed in the next section. An assessment of the applicability of these studies to tropical cyclones is one of the two important aims of this article detailed in section 1.3.

\subsection{The Turner-Lilly-Morton ideas}

In general, there are two fundamental requirements for vortex amplification: a source of rotation and some forcing mechanism to concentrate the rotation. An example is one of the laboratory experiments described by Turner and Lilly (1963) in which a vortex was produced in water contained in a rotating cylinder by releasing bubbles from a thin tube along the upper part of the rotation axis. A sketch of the flow configuration is shown in Figure 1. The drag on the fluid exerted by the ascending bubbles generates a secondary circulation in the water, producing convergence in the region below the source of bubbles. Except in a shallow boundary layer near the lower boundary, converging rings of fluid conserve their angular momentum and spin faster. The ultimate degree of amplification of the angular velocity depends on how far rings of fluid can be drawn inwards and on the background rotation rate, factors that are not independent.

How far rings of fluid can be drawn inwards depends, inter alia, on the forcing strength, i.e. on the bubbling rate. If the forcing is sufficiently large for a given rotation rate, rings of fluid may be drawn in to relatively small radii before the centrifugal force $\left(v^{2} / r\right)$ and Coriolis force $(2 \Omega v)$ opposing the inward motion balance the radial pressure gradient induced by the bubbles. It is this pressure gradient that drives the rings of fluid inwards (right of Figure 1). Here $v$ is the azimuthal velocity component, $r$ is the radius from the rotation axis and $\Omega$ is the background rotation rate. If the forcing is comparatively weak, or if the rotation rate is sufficiently strong, this balance may be achieved before the radial displacement is very large, so that a significant amplification of the background rotation will not be achieved. Of course, if there is no background rotation, there will be no amplification, and if the background rotation is very weak, the centrifugal and/or Coriolis forces never become large enough to balance the radial pressure gradient, except possibly at large radii from the source of bubbles. Note that these arguments emphasize the unbalanced wind adjustment from the instant that the bubbling is turned on.

Clearly, one would expect the existence of an optimum forcing strength to produce the maximum amplification

boundary layer in his intensifying vortex, but that this inflow is implicit in the absolute angular momentum coordinate used. Thus, despite the fact that the air is rising along absolute angular momentum surfaces and that these surfaces flare outwards with height, these surfaces, themselves, are moving inwards, so that air parcel trajectories have a net inward component. Nevertheless, Emanuel's (1997) theory assumes gradient wind balance in the boundary layer (see first two complete paragraph on p. 1018 of Emanuel, 1997). Because of this physical inconsistency and the ad hoc nature of this model through the introduction of the $\beta$-parameter (details in Montgomery et al., 2009, footnote on p. 1698), we do not regard this as a satisfactory theoretical description of the spin-up process.

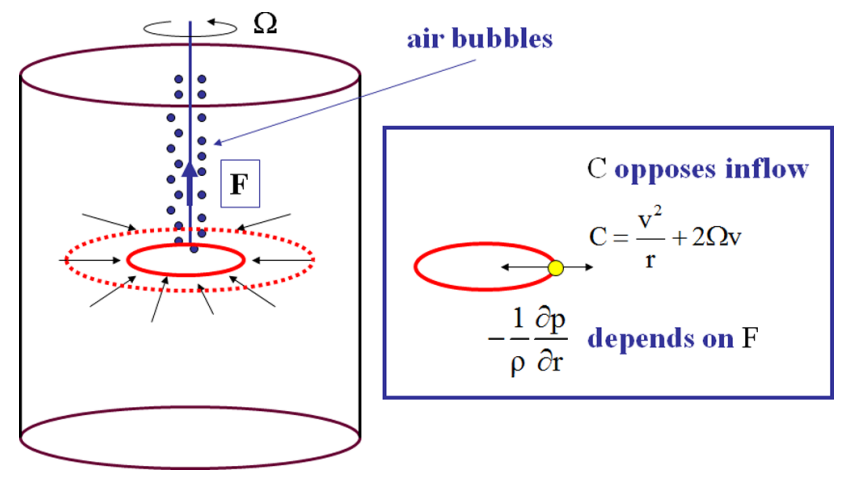

Figure 1. Schematic of flow configuration in the Turner and Lilly experiment. The right side indicates the principal forces per unit mass acting on an air parcel in the radial direction: an inward-directed pressure gradient force $-(1 / \rho)(\partial p / \partial r)$ and an outward force $C$, the sum of the centrifugal force $\left(v^{2} / r\right)$ and Coriolis force $(2 \Omega v)$. This figure is available in colour online at wileyonlinelibrary.com/journal/qj

of the angular velocity for a given strength of background rotation, or an optimum background rotation rate for a given forcing strength. These ideas were explained eloquently by Morton (1966) and demonstrated in related numerical experiments by Smith and Leslie $(1976,1978)$. The dustdevil-like vortices investigated in Smith and Leslie (1976) are driven by the buoyancy arising from thermal heating of the surface, but it turns out that the high-rotation-rate regime produces warm-cored, two-cell vortices reminiscent of tropical cyclones. A question arises: to what extent do the above ideas apply to tropical cyclones?

\subsection{Application to tropical cyclones}

One difference between the foregoing laboratory and numerical experiments and a tropical-cyclone model is that, in the latter, one does not have the flexibility to independently vary the rotation rate and the strength of the forcing. Indeed, we will show in section 6 that the 'effective forcing strength' varies with the intensity. However, one can control the effective background rotation by varying the Coriolis parameter, $f$. Another potential difference is the role of the boundary layer in tropicalcyclone spin-up as discussed above. Whether the boundary layer is of comparable importance in the spin-up of tall narrow vortices of the type discussed above is unknown to us, although Turner's (1966) approximate theoretical description of his laboratory vortex recognised the strong constraint imposed by the boundary layer in the steady problem. A third difference is the fact that the azimuthally averaged component of flow in a tropical cyclone is close to gradient wind balance except in the boundary layer and the outflow layer in the upper troposphere (Willoughby, 1979). In contrast, as noted above, the Turner-Lilly-Morton (TLM) ideas highlight the temporal adjustment to gradient wind balance.

Recent studies (Nguyen et al., 2008; Shin and Smith, 2008) have shown that tropical-cyclone intensification is intrinsically a three-dimensional process in which deep rotating convective clouds, or 'vortical hot towers' (VHTs), play a central role. In the non-hydrostatic model of Nguyen et al., these towers have local buoyancy relative to the azimuthally averaged density field and collectively mimic a positive, azimuthal-mean, diabatic heating rate that drives a quasi-balanced transverse circulation on the 
system scale. The radial gradient of this heating rate acts to drive an azimuthal-mean secondary circulation with convergence in the lower troposphere. This convergence enhances the azimuthal-mean vorticity and contributes to the aggregation of convectively generated cyclonic vorticity, both of which are key features of the spin-up of the mean vortex (Montgomery et al., 2006, 2009; Bui et al., 2009). Despite the important role of the VHTs, one can usefully apply arguments based on the evolution of the axisymmetric mean fields as in Smith et al. (2009) and Bui et al. (2009). In this spirit, and to retain maximum simplicity, we confine our attention here to the axisymmetric aspects of vortex spin-up.

The present paper investigates the effects of the ambient rotation rate on tropical-cyclone evolution. In particular, we seek to explore two questions:

- How does the ambient rotation rate characterized by the Coriolis parameter influence the intensity and size of a tropical cyclone?

- Can this influence be interpreted in terms of the TLM ideas?

The article is organized as follows. The model used and the details of the numerical experiments carried out are described in sections 2 and 3. The results of these experiments are presented in sections 4 and 8 . In section 5 we demonstrate the importance of the boundary layer in the spin-up of the inner core of the vortex and in section 6 we discuss the concept of 'effective forcing' for a tropical cyclone. An appraisal of the TLM ideas is given in section 7 . A discussion of the results is presented in section 9 and the conclusions are given in section 10 .

\section{The numerical model}

The model used for the calculations is the axisymmetric version of the minimal three-layer hurricane model described by Zhu etal. (2001), developed by Nguyen et al. (2002). It is a hydrostatic model formulated in $\sigma$-coordinates on an $f$-plane, where $\sigma=\left(p-p_{\text {top }}\right) / p^{*}$, $p^{*}=p_{\mathrm{s}}-p_{\text {top }}, p_{\mathrm{s}}$ and $p_{\text {top }}$ are the surface and top pressures, respectively, $p_{\text {top }}$ is a constant (taken here to be $100 \mathrm{mb}$ ), and $f$ is the Coriolis parameter. The lower interface $\sigma$ level is $8 / 9$ and the upper one is $3 / 9$. The model equations are identical to those detailed in Nguyen et al. (2002). The upper and lower boundary conditions require that $\dot{\sigma}=0$ at $\sigma=0$ and $\sigma=1$, where $\dot{\sigma}=\mathrm{D} \sigma / \mathrm{D} t$ is the 'vertical' $\sigma$-velocity and $\mathrm{D} / \mathrm{D} t$ is the material derivative. The model has a $10 \mathrm{~km}$ radial grid spacing and the radial domain size extends to $1000 \mathrm{~km}$. The integration time step is $6 \mathrm{~s}$. Latent heat release in deep cumulus clouds is represented by a parametrization scheme proposed by Arakawa (1969). The scheme is a type of mass flux scheme in which the subgrid-scale mass flux is determined by assuming that deep convection tends to remove any conditional instability on a prescribed time-scale. This time-scale is typically on the order of an hour. The removal of instability is accomplished by relaxing the moist static energy of the upper layer towards that of the boundary layer on the assumed time-scale. This scheme is complemented by a simple explicit scheme that is implemented where there is condensation on the grid-scale. The two schemes are complementary in the sense that once a grid column saturates, the convection parametrization

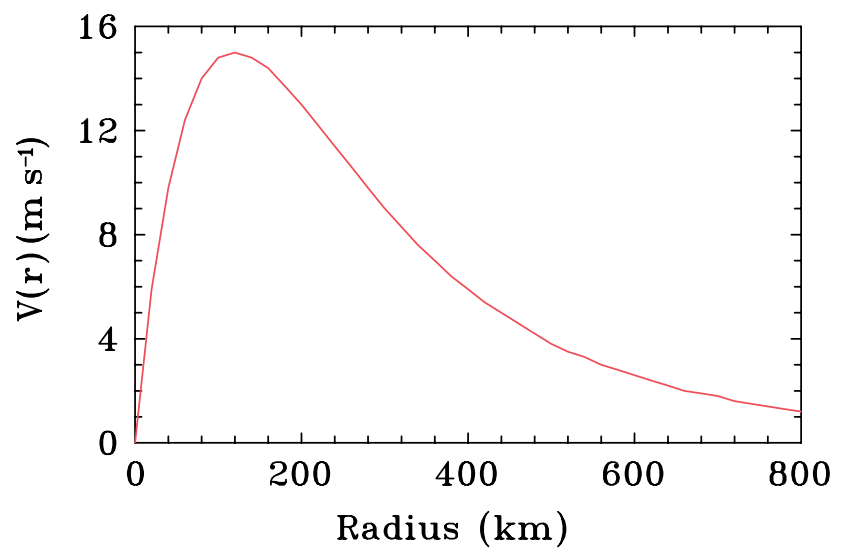

Figure 2. Radial profile of tangential wind in the lower layer for the initial vortex. This figure is available in colour online at wileyonlinelibrary.com/journal/qj

scheme turns off in that grid column. Zhu et al. (2001) give further details.

\section{The calculations}

Two sets of calculations are carried out to address the questions posed in section 1.3. In the first set, we investigate the evolution of a vortex growing from the same initial baroclinic vortex in an otherwise quiescent environment with different levels of background rotation characterized by $f$. In particular, we investigate the dependence of the vortex intensity, characterized by the maximum tangential wind speed, and the vortex size, characterized by the radius of gale-force winds, on the background rotation strength, measured by the Coriolis parameter. Taking a reference latitude of $20^{\circ} \mathrm{N}$ and denoting the corresponding Coriolis parameter as $f_{0}$, we carried out calculations for ten values of $f: f=0,0.1 f_{0}, 0.25 f_{0}, 0.5 f_{0}, 0.75 f_{0}, f_{0}, 1.25 f_{0}, 1.5 f_{0}, 1.75 f_{0}$, and $1.9 f_{0}$, corresponding to the Equator and latitudes $2.4^{\circ} \mathrm{N}$, $4.9^{\circ} \mathrm{N}, 9.8^{\circ} \mathrm{N}, 14.9^{\circ} \mathrm{N}, 20.0^{\circ} \mathrm{N}, 25.3^{\circ} \mathrm{N}, 30.9^{\circ} \mathrm{N}, 36.8^{\circ} \mathrm{N}$ and $40.5^{\circ} \mathrm{N}$.

In order to suppress the implicit dependence of the effective forcing on the vortex intensity, a second set of calculations is carried out in which moist processes are absent and an approximately equivalent diabatic heating rate is prescribed. The heating rate has a radial distribution and magnitude typical of that diagnosed from the first set in the mature stage of the vortex. The strength of the heating is increased gradually over a $24 \mathrm{~h}$ period and then held fixed to minimize the generation of unrealistic inertia-gravity waves.

The initial radial profile of tangential wind in the lower layer is shown in Figure 2. The profiles in the middle and upper layers are the same, but their amplitudes are multiplied by factors of 0.9 and 0.3 , respectively.

The two sets of experiments are discussed in sections 4 and 8 , respectively.

\section{Dependence on the Coriolis parameter}

Figure 3 shows time series of maximum tangential wind speed, $v_{\max }$, in the middle and lower layers in the set of ten moist calculations with different values of $f$ and with the initial vortex profile in Figure 2. It shows also the radius, $r_{\text {gales}}$, at which the tangential wind speed reaches gale force 

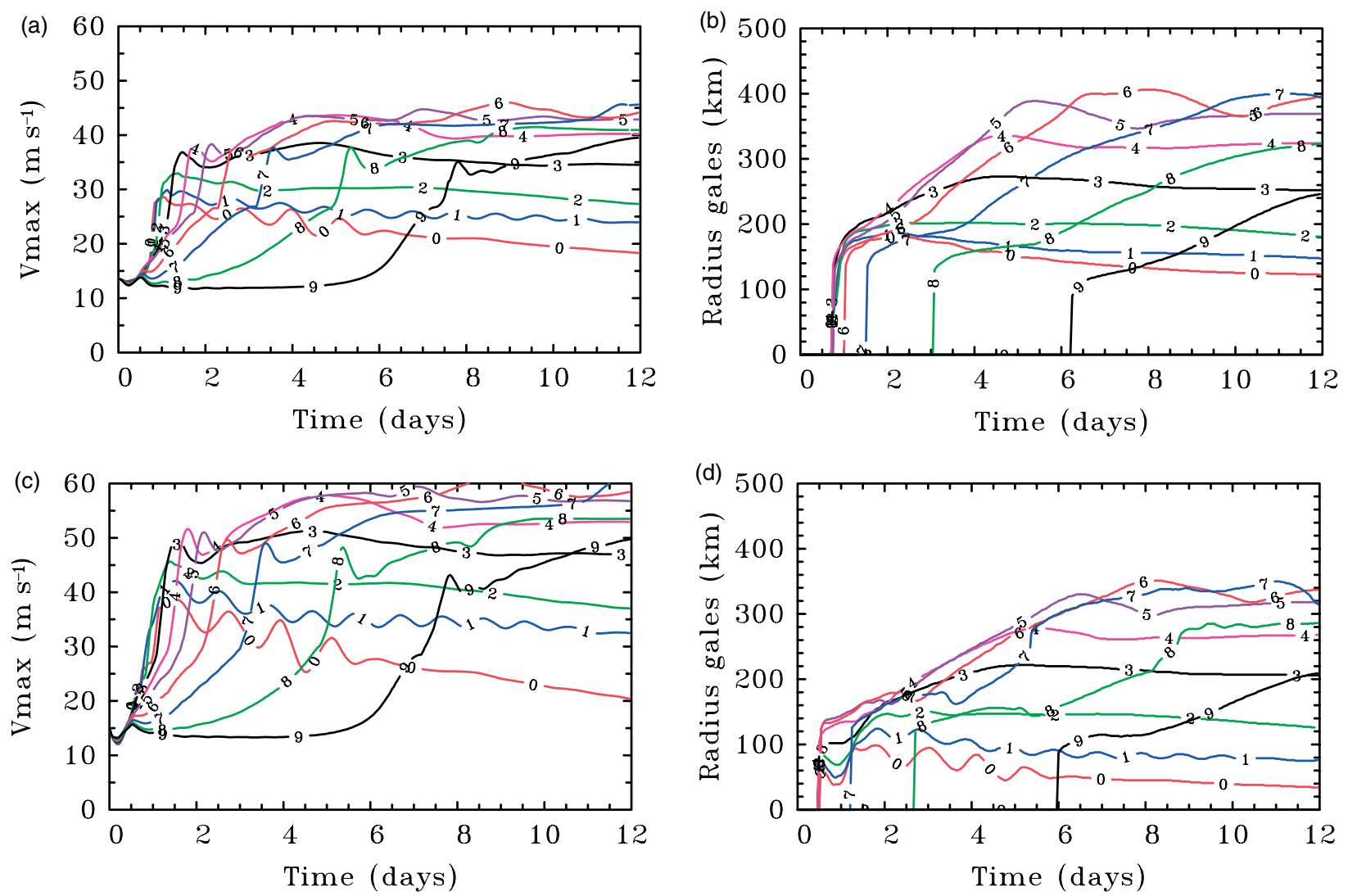

Figure 3. Time series of ( $a, c)$ maximum tangential wind speed and (b, d) radius of gale-force winds in ( $a, b)$ the middle layer and in (c, d) the lower layer, in the set of calculations with different values of $f$. Curves labelled $0-9$ respectively denote the sequence of $f$ values: $0,0.1 f_{0}, 0.25 f_{0}, 0.5 f_{0}, 0.75 f_{0}$, $1.0 f_{0}, 1.25 f_{0}, 1.5 f_{0}, 1.75 f_{0}$ and $1.9 f_{0}$. This figure is available in colour online at wileyonlinelibrary.com/journal/qj

$\left(17 \mathrm{~m} \mathrm{~s}^{-1}\right)$ in each layer. The dependence of $v_{\max }$ and $r_{\text {gales }}$ on $f$ are discussed in the next two subsections.

\subsection{Vortex intensity}

There is a clear dependence of $v_{\max }$ on $f$, with both the onset time of rapid intensification increasing and the intensification rate decreasing slightly as the rotational constraint (i.e. $f$ ) increases. The low-latitude vortices $\left(f \leq 0.25 f_{0}\right)$ show the earliest and most rapid intensification, but their intensity after a few days is less than those at higher latitudes. The intensity of the two lowest-latitude vortices shows considerable oscillatory behaviour during the decay phase. Significantly, the largest values of $v_{\max }$ occur in the lowest layer, which is the layer influenced by surface friction. This result is consistent with the findings of Zhang et al. (2001), Nguyen et al. (2002) and Smith et al. (2009), who showed that the spin-up of the vortex core occurs within the frictional boundary layer.

The intensity after twelve days, characterized by $v_{\max }$ in either the boundary layer or middle layer, is summarized in Figure 4(a), which shows $v_{\max }$ as a function of $f$ in both layers. The most intense vortex is that which develops in an environment with background rotation $f=1.5 f_{0}$, i.e. there exists an optimum background rotation for maximum intensity. At first sight, this result would seem to be consistent with those of the Turner-Lilly laboratory experiments, but as we will show in section 7 , care is required in making a direct analogy with the experiments. It is consistent also with the results of DeMaria and Pickle (1988, p. 1552), who found that the intensity at $20^{\circ}$ latitude $\left(57 \mathrm{~m} \mathrm{~s}^{-1}\right)$ was marginally stronger (by $2 \mathrm{~m} \mathrm{~s}^{-1}$ ) than that at $10^{\circ}$ latitude and decreased to $51 \mathrm{~m} \mathrm{~s}^{-1}$ at $30^{\circ}$ and $46 \mathrm{~m} \mathrm{~s}^{-1}$ at $40^{\circ}$ latitude.

\subsection{Vortex size}

Referring to Figure 3(b), it is seen that, in the middle layer, for the smallest values of $f\left(0 \leq 0.5 f_{0}\right.$ : curves $\left.0-3\right)$, $r_{\text {gales }}$ increases rapidly to a maximum and thereafter slowly declines. For larger values of $f$ (curves 4-9), $r_{\text {gales }}$ increases steadily with time and finally levels out, or it reaches a peak and then declines a little, in some cases fluctuating (e.g. curves 5 and 6), before levelling out. In the lower layer, the behaviour is broadly similar (Figure $3(\mathrm{~d})$ ). Note that, in this layer, $r_{\text {gales }}$ is generally smaller than in the middle layer because the winds at large radii are subgradient on account of friction.

Figure 4(b) shows $r_{\text {gales }}$ as a function of $f$ in the middle and lower layers after twelve days. The results indicate the existence of an optimum background rotation strength to obtain the largest vortex as characterized by $r_{\text {gales. }}$. This finding is contrary to that of DeMaria and Pickle (1988), who found that the vortex size increases monotonically with latitude. Nevertheless, it is pertinent to ask also whether our result can be interpreted in terms of those from the Turner-Lilly laboratory experiments. We shall show in section 7 that this is not the case, but in doing so we raise a number of issues that we believe to be important in tropical-cyclone dynamics.

DeMaria and Pickle noted that their finding concerning vortex size ' ... appears to be related to the radial positioning of the diabatic heating'. They argued (p. 1551) that the 

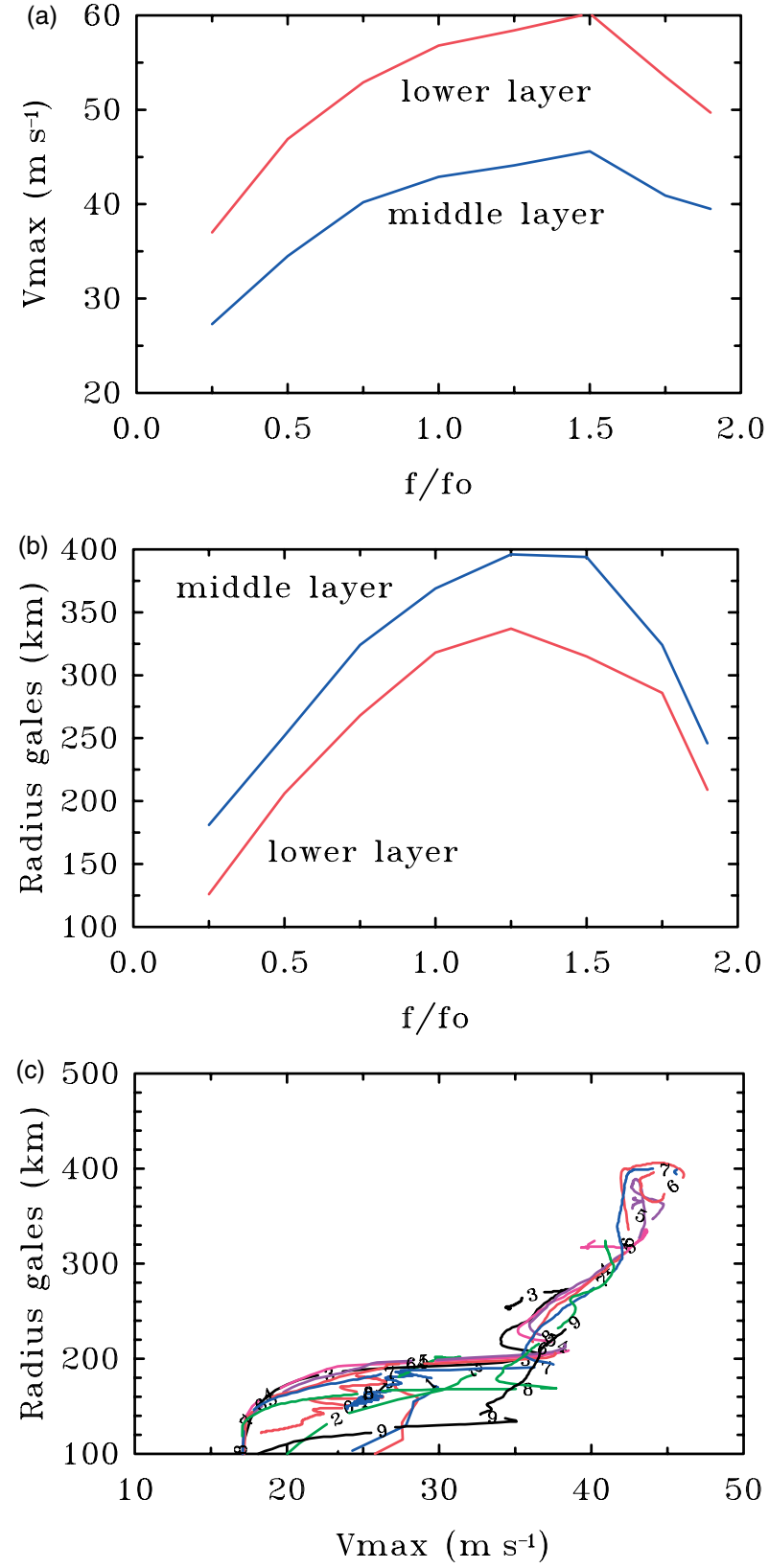

Figure 4. Variation of (a) the maximum tangential wind, and (b) radius of gales (based on this wind component) with the Coriolis parameter in the lower and middle layers at $12 \mathrm{~d}$ in the calculations described in section 4 . (c) shows the radius of gales in the middle layer as a function of intensity, measured by the instantaneous value of $v_{\max }$ during the $12 \mathrm{~d}$ life cycle of the ten variable- $f$ calculations, with curves labelled as in Figure 3. This figure is available in colour online at wileyonlinelibrary.com/journal/qj

boundary-layer convergence and thus the diabatic heating are closer to the centre as the storm latitude is decreased. Since the diabatic heating does not vary rapidly as a function of latitude, 'the same diabatic heating occurs at smaller radii at low latitudes resulting in smaller storms'. A difficulty with this interpretation is that the diabatically induced secondary circulation is related to the radial gradient of diabatic heating rate rather than the heating rate alone (section 6).

\subsection{Intensity versus size}

Figure 4(c) shows $r_{\text {gales }}$ as a function of $v_{\max }$ at all times during the foregoing calculations. Note that there is not a one-to-one correspondence between the intensity of a vortex
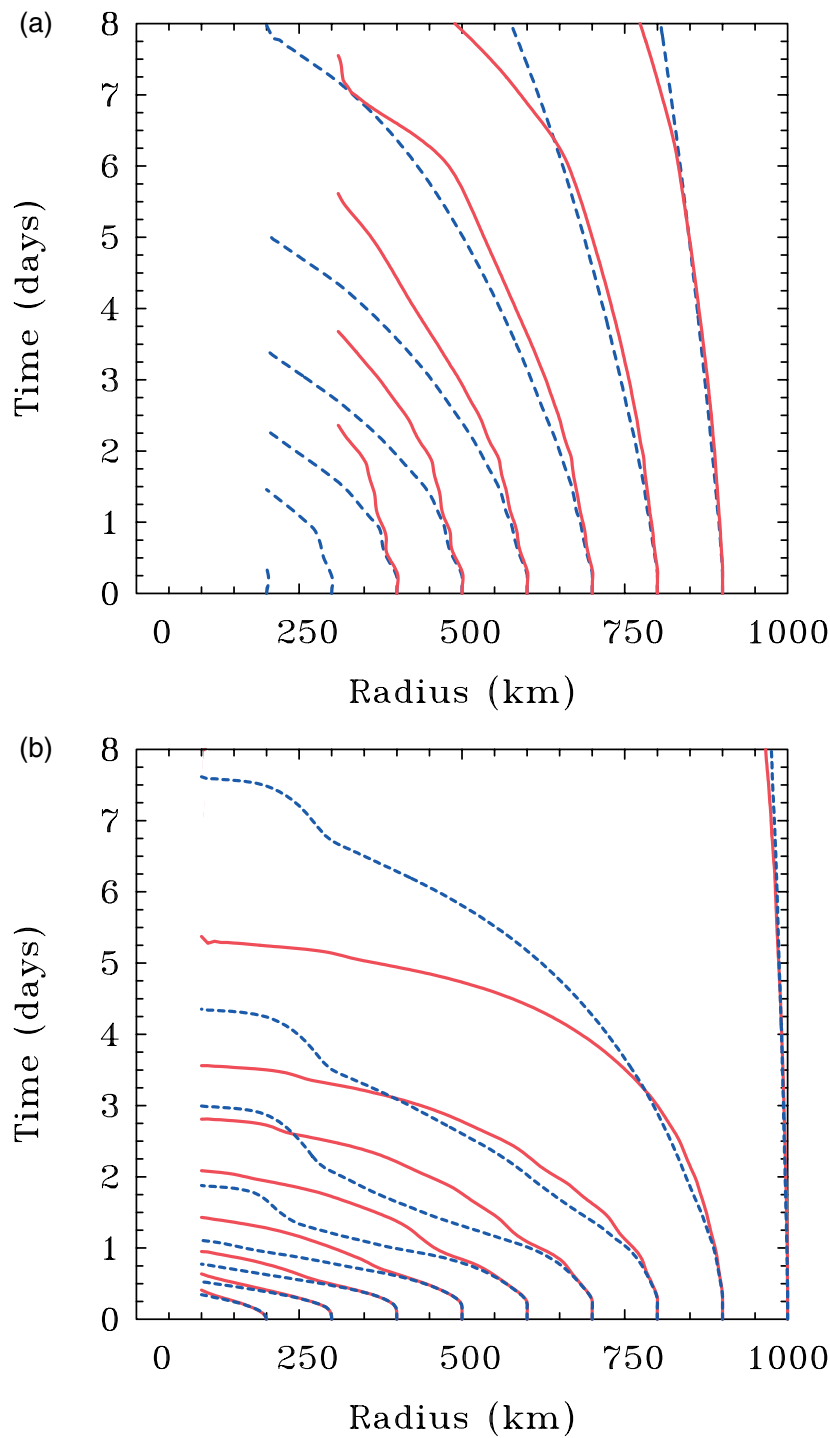

Figure 5. Time-radius plots of the trajectories in (a) the middle layer and (b) the boundary layer in the calculations with $f=0.25 f_{0}$ (dashed lines) and $f=f_{0}$ (solid lines). This figure is available in colour online at wileyonlinelibrary.com/journal/qj

and its size (as measured by $r_{\text {gales }}$ ) during the entire period of development. This lack of a relationship is in line with the observational study of Weatherford and Gray (1988) and a possible reason for it is discussed by Smith et al. (2009). However, it is evident from the figure that, for the vortices studied here, which all start with the same modest-sized initial vortex, there is an approximate linear increase in $r_{\text {gales }}$ with $v_{\max }$ for $v_{\max }>35 \mathrm{~m} \mathrm{~s}^{-1}$.

\subsection{Trajectories}

The TLM ideas invoke the ability of a given forcing strength to produce radial displacements of rings of air. Thus it is pertinent to examine the air-parcel trajectories. Figure 5 shows time-radius plots of a passive tracer in the lower and middle layers in the calculations with $f=0.25 f_{0}$ and $f=f_{0}$. The tracer evolution is calculated by solving the radial advection equation in each layer with the initial value of the tracer equal to the initial radius. Details of the calculation are given in Appendix A.1. The tracer contours correspond approximately with the trajectories of air columns in a layer 

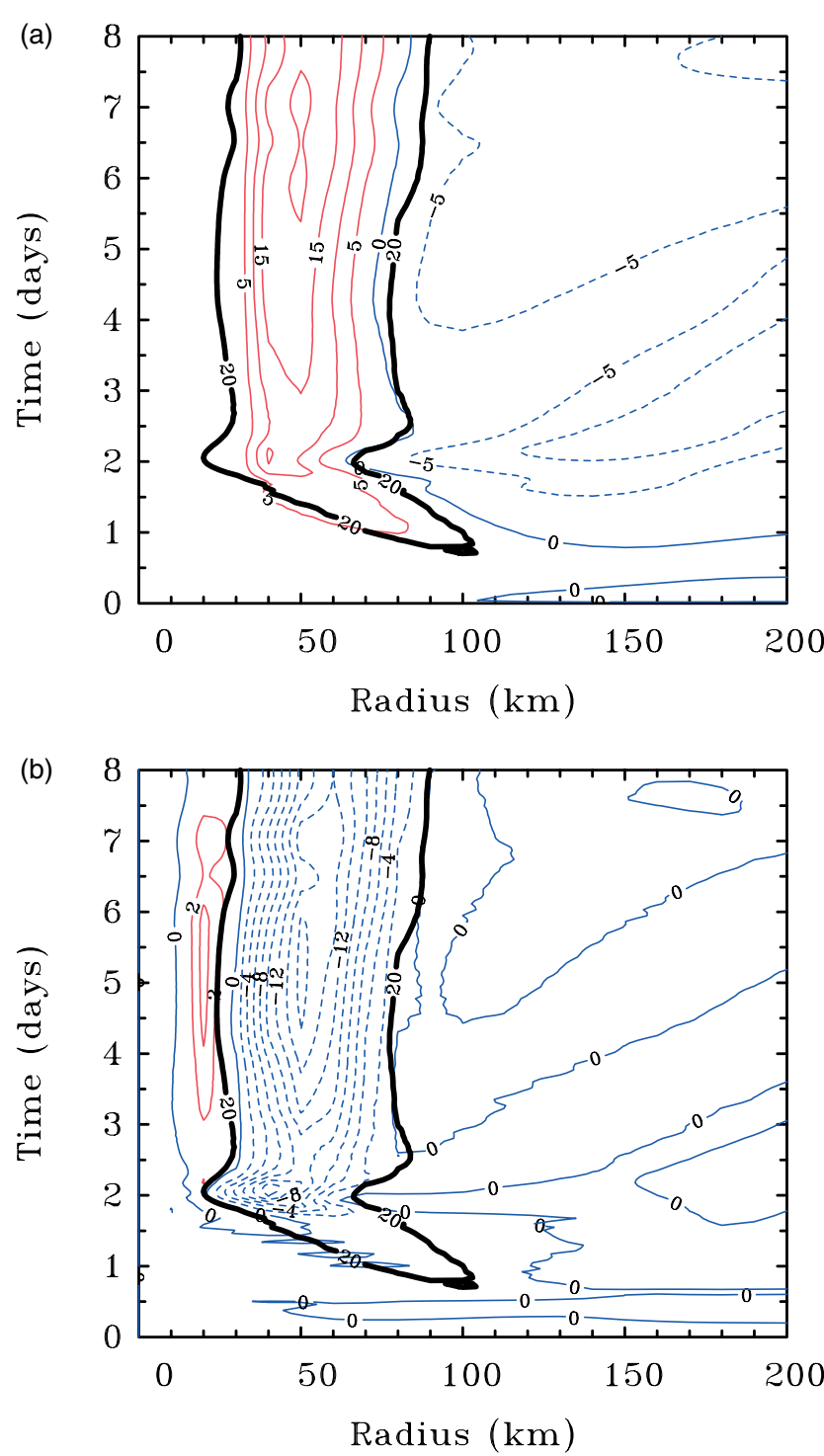

Figure 6. Time-radius plots of (a) the difference between the tangentia wind speed in the boundary layer and the middle layer (contour interval $5 \mathrm{~m} \mathrm{~s}^{-1}$ ), and (b) the radial flux of absolute vorticity in the middle layer in the moist calculation of section 4 with $f=f_{0}$ (contour interval $2 \mathrm{~m} \mathrm{~s}^{-1} \mathrm{~h}^{-1}$, with positive contours solid and negative contours dashed). The bold black curve is the $20 \mathrm{~cm} \mathrm{~s}^{-1}$ contour of vertical velocity from the boundary layer to the middle layer. This figure is available in colour online at wileyonlinelibrary.com/journal/qj

except in regions where there is appreciable vertical exchange of mass between layers. Therefore, we have not shown contours in the inner region where there is a substantial flow of air entering from the boundary layer or exiting into the upper layer.

The patterns of tracer movement in the middle layer are consistent with the idea discussed in section 1.2 that the inward radial displacements of air columns should be larger in the case with smaller $f$, although this argument assumes that the forcing strength does not change appreciably between these calculations. In fact, it will be shown in section 6 that the forcing strength is mostly larger in the case with $f=f_{0}$, so that there are two competing effects.

It is clear from Figure 5 that the radial displacements in each experiment are very much larger in the lower layer (where friction is important) than in the middle layer. As discussed in Smith et al. (2009), it is for this reason that the largest tangential wind component occurs in the boundary layer (cf. Figures 3(a, c)).

\section{Spin-up in the model}

To illustrate the importance of boundary-layer spin-up in the present model, we show in Figure 6 time-radius plots of the difference between the tangential wind component in the boundary layer and the middle layer and of the radial flux of absolute vorticity in the middle layer, $-u(\zeta+f)$, where $u$ is the radial velocity component and $\zeta$ is the vertical component of relative vorticity. The two panels show also where there is vertical motion and, in particular, strong upward motion. It is significant that the strong upward motion from the boundary layer to the middle layer coincides with the region where the tangential wind speed is larger in the boundary layer. Thus, in this region, the middle layer is being fed by angular-momentum-enriched air. In the same annular region, there is a strong outward flux of absolute vorticity in the middle layer, equivalent to a negative advection of absolute angular momentum, which, by itself would rapidly spin down the flow. Clearly, the spin-up of the middle layer in this annular region is a result of the upward transfer of higher values of absolute angular momentum from the boundary layer and not from the radial convergence of absolute angular momentum in the middle layer, supporting the ideas articulated by Nguyen et al. (2002), Smith et al. (2009) and Smith and Montgomery (2010). It is worth pointing out that this process of spin-up cannot occur in time-dependent models that directly or tacitly assume gradient wind balance in the boundary layer such as those of Ooyama (1969), Emanuel (1997), Frisius (2006) and Wirth and Dunkerton (2006).

Outside a radius of $\approx 80 \mathrm{~km}$ in Figure $6(\mathrm{~b})$, there is an inward vorticity flux so that the spin-up there is associated with the radial convergence of absolute angular momentum in that layer.

Together, the foregoing results are consistent with the two mechanisms of spin-up discussed in section 1.1.

\section{Effective forcing}

At this point it is appropriate to examine the nature of the 'effective forcing' in the experiments detailed in section 4 and how this forcing might be quantified. In balance models in which gradient and hydrostatic balance is assumed, the forcing of the secondary circulation is related, inter alia, to the radial gradient, $\partial \dot{\theta} / \partial r$, of the diabatic heating rate, $\dot{\theta}$ (Shapiro and Willoughby, 1982; Bui et al., 2009). The overturning circulation outside the eye is characterized by the maximum magnitude of the negative radial gradient of diabatic heating that lies radially outside the heating maximum. Since balance dynamics is found to be a good first approximation above the friction layer (e.g. Willoughby, 1990; Bui et al., 2009), it would seem reasonable to take the maximum of $-\partial \dot{\theta} / \partial r$ as a suitable measure for the effective forcing, at least to a first approximation. To this end, the heating rate and its radial gradient have been diagnosed from the model output (Appendix A.2).

Figure 7 shows the time evolution of this maximum for eight of the ten calculations described above (the curves for $f=0$ and $f=0.1 f_{0}$ are omitted as they have significant oscillations that clutter the diagram). Comparing Figures 3 and 7 , it is seen that the maximum of $-\partial \dot{\theta} / \partial r$ increases 


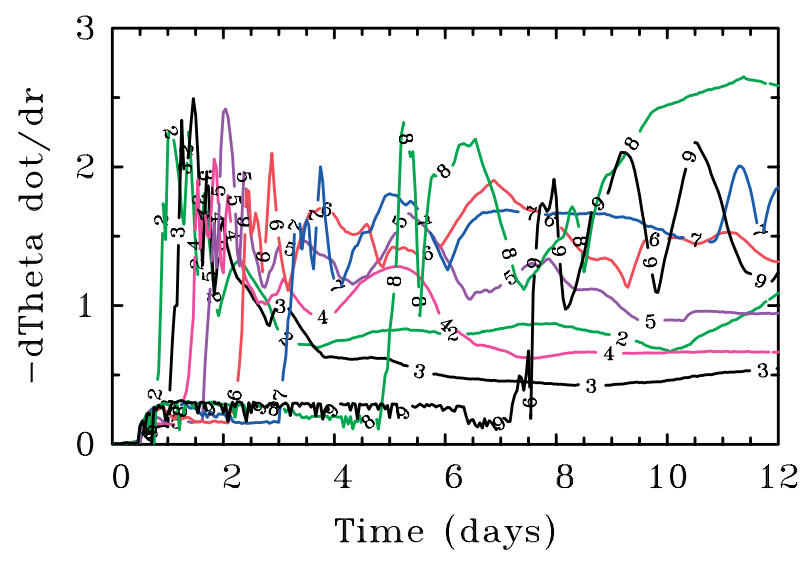

Figure 7. Time series of the maximum magnitude of the negative radial gradient of the diabatic heating rate $\left(\mathrm{K} \mathrm{h}^{-1} \mathrm{~km}^{-1}\right)$ in the middle layer in the set of calculations with different values of $f \geq 0.25 f_{0}$. Curve numbering is as in Figure 3. This figure is available in colour online at wileyonlinelibrary.com/journal/qj

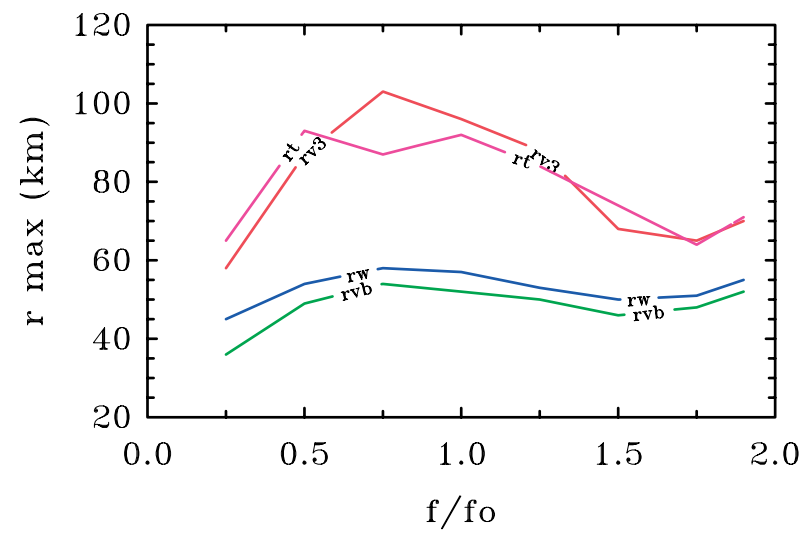

Figure 8. Variation of the radii of maximum radial gradient of diabatic heating rate (curve rt), maximum vertical velocity (curve rw) from the boundary layer to the middle layer, and maximum tangential wind speed in the middle layer (curve rv3) and boundary layer (curve rvb), with the Coriolis parameter at $12 \mathrm{~d}$ in the calculations described in section 4 . This figure is available in colour online at wileyonlinelibrary.com/journal/qj

sharply at the time of onset of rapid intensification. As shown by Zhu et al. (2001), the onset of rapid intensification is accompanied by saturation on the grid-scale in the innercore region. At grid points where saturation occurs, the parametrized convection scheme switches off and the explicit scheme for latent heat release takes over. For each value of $f$, the maximum gradient of the heating rate increases sharply at first and then declines, but with significant oscillations. As in calculations with more complex models (e.g. Rotunno and Emanuel, 1987; Persing and Montgomery, 2003; Nguyen et al., 2008), these oscillations are associated with pulsations in the explicit convection, which, in turn, are accompanied by inertia-gravity waves that the convection induces. After about day 10, the 'effective forcing' is larger in the calculations at the higher latitudes $\left(f \geq 1.25 f_{0}\right.$, curves 6-9) than those at latitudes with $f \leq f_{0}$.

Following DeMaria and Pickle's (1988) finding that the boundary-layer convergence and thus the diabatic heating occur much closer to the storm centre as the latitude is decreased, we were motivated to investigate the dependence of the radius of the maximum magnitude of the negative radial gradient of diabatic heating rate, $r \max (-\partial \dot{\theta} / \partial r)$, in the middle layer and to compare this with the radius of
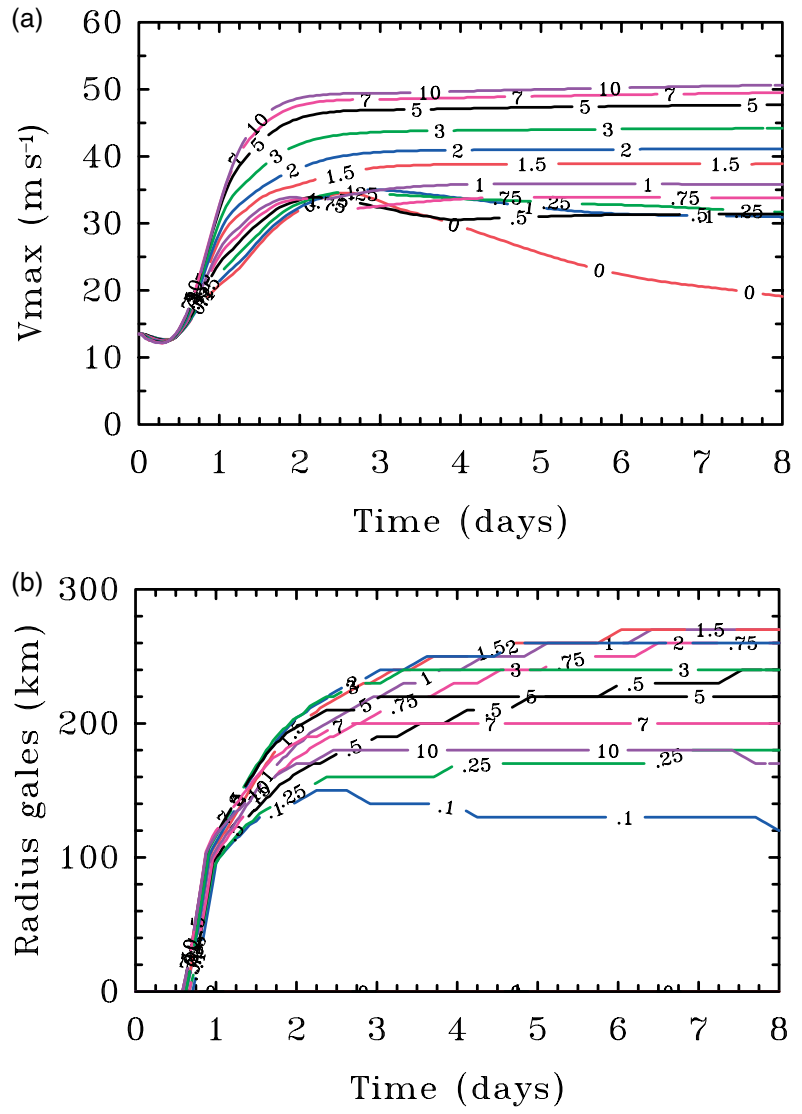

Figure 9. Time series of (a) the maximum tangential wind, and (b) the radius of gale-force winds in the middle layer in the set of calculations with fixed forcing and different values of $f: 0,0.1 f_{0}, 0.25 f_{0}, 0.5 f_{0}$, $0.75 f_{0}, f_{0}, 1.5 f_{0}, 2 f_{0}, 3 f_{0}, 5 f_{0}, 7 f_{0}$ and $10 f_{0}$. Curves are labelled with the corresponding multiple of $f_{0}$. This figure is available in colour online at wileyonlinelibrary.com/journal/qj

maximum vertical velocity, $r \max (w)$, from the lower layer to the middle layer and the radii of maximum tangential wind speed in the lower and middle layers, $r \max \left(v_{\mathrm{b}}\right)$ and $r \max \left(v_{3}\right)$, respectively. These radii are shown in Figure 8 after twelve days of integration. It is seen that there is a reasonable correlation between $r \max (-\partial \dot{\theta} / \partial r)$ and $r \max (w)$ with the former lying between 10 and $40 \mathrm{~km}$ outside the latter, which is one to four times the horizontal grid spacing in the model ${ }^{9}$. Consistent with the absolute angular momentum surfaces sloping outwards above the boundary layer, $\operatorname{rmax}\left(v_{3}\right)$ is significantly larger than $r \max \left(v_{\mathrm{b}}\right)$, but note that $r \max \left(v_{\mathrm{b}}\right)$ and $r \max (w)$ follow each other, with $r \max (w)$ lying outside of $r \max \left(v_{\mathrm{b}}\right)$, but within $10 \mathrm{~km}$ (i.e. one grid interval) of it. The broadest vortex, based on either $r \max \left(v_{\mathrm{b}}\right)$ or $r \max \left(v_{3}\right)$, occurs for $f=0.75 f_{0}$, but this is not the most intense vortex. Indeed, comparison of Figures $3(a, c)$ with Figure 8 shows that there is not an obvious relationship between the maximum tangential wind speed and the radius at which it occurs in either layer.

\section{An appraisal of the TLM ideas}

We examine now the question: can the foregoing results be interpreted in terms of the TLM ideas discussed in section $1.2 ?$

To produce smoother fields, the radius of the maxima of particular quantities were obtained to within a kilometre by spline interpolation. 


\subsection{Relevance to intensity}

At first sight, the dependence of $v_{\max }$ on $f$ discussed in section 4.1 is suggestive that the TLM ideas might apply in a broad sense. Moreover, as shown in section 4.4, the patterns of tracer movement in the middle layer would be consistent with the expectation based on inertial stability considerations that the inward radial displacements of air columns should be larger in the case with smaller $f$, at least as long as the forcing strength does not change appreciably between these calculations. However, we showed in section 6 that the forcing strength is mostly larger in the case with the larger rotation rate, at least for the two values of $f$ shown in Figure 5. Thus there are two competing effects: while the forcing tries to draw air columns inwards in the middle layer, the inertial stability resists these displacements. It does not appear possible to foresee the outcome of these competing effects in a simple way and one needs to do an explicit calculation.

A further issue is that, as noted in section 1.2, the optimum amplification in the Turner-Lilly experiment refers to the fractional increase in angular velocity and not directly to intensity, which is the quantity of interest here, and indeed to tropical-cyclone forecasters.

Another important limitation is the fact that in a tropical cyclone, the spin-up of the inner-core winds occurs in the boundary layer (Emanuel, 1997; Zhang et al., 2001; Smith et al., 2009; Sanger, 2011). The results in section 5 show the same feature in that the intensity in the middle layer is determined by the vertical advection of air from the boundary layer and is not a result of the convergence of absolute angular momentum in the middle layer as envisaged by the conventional view of tropical-cyclone intensification (Ooyama, 1969, 1982 (p. 374); Shapiro and Willoughby, 1982; Smith et al., 2009; Montgomery and Smith, 2011).

In the light of these findings, we have not plotted a curve showing maximum angular velocity in the middle layer as a function of $f$ because the TLM ideas apply only to radial advection above the boundary layer.

The breakdown of the TLM ideas in the boundary layer are illustrated by the patterns of tracer movement in the lower layer shown in Figure 5(b). In this layer, the degree of inertial stability is overshadowed by the effects of friction, which, beyond a certain radius, ensure that the net radial force is inward, irrespective of the degree of inertial stability as normally calculated. Thus, because of friction, there is no inertial resistance to radial displacements in this layer" ${ }^{\|}$

\subsection{Relevance to size}

We enquire now whether the TLM ideas provide an interpretation of the optimum value of background rotation to produce the largest vortex as found in section 4.2. One could argue that, at zero ambient rotation, the only source of angular momentum is that of the initial vortex and this angular momentum is progressively removed by surface friction. As long as deep convection is maintained, there has to be a period of intensification and broadening in this case. Ultimately, however, the angular momentum in the lower and middle troposphere will steadily diminish and the

"The concept of inertial stability is normally presented for a situation in which a swirling flow in gradient wind balance with no radial motion is symmetrically perturbed (Rayleigh, 1917; Charney, 1973).
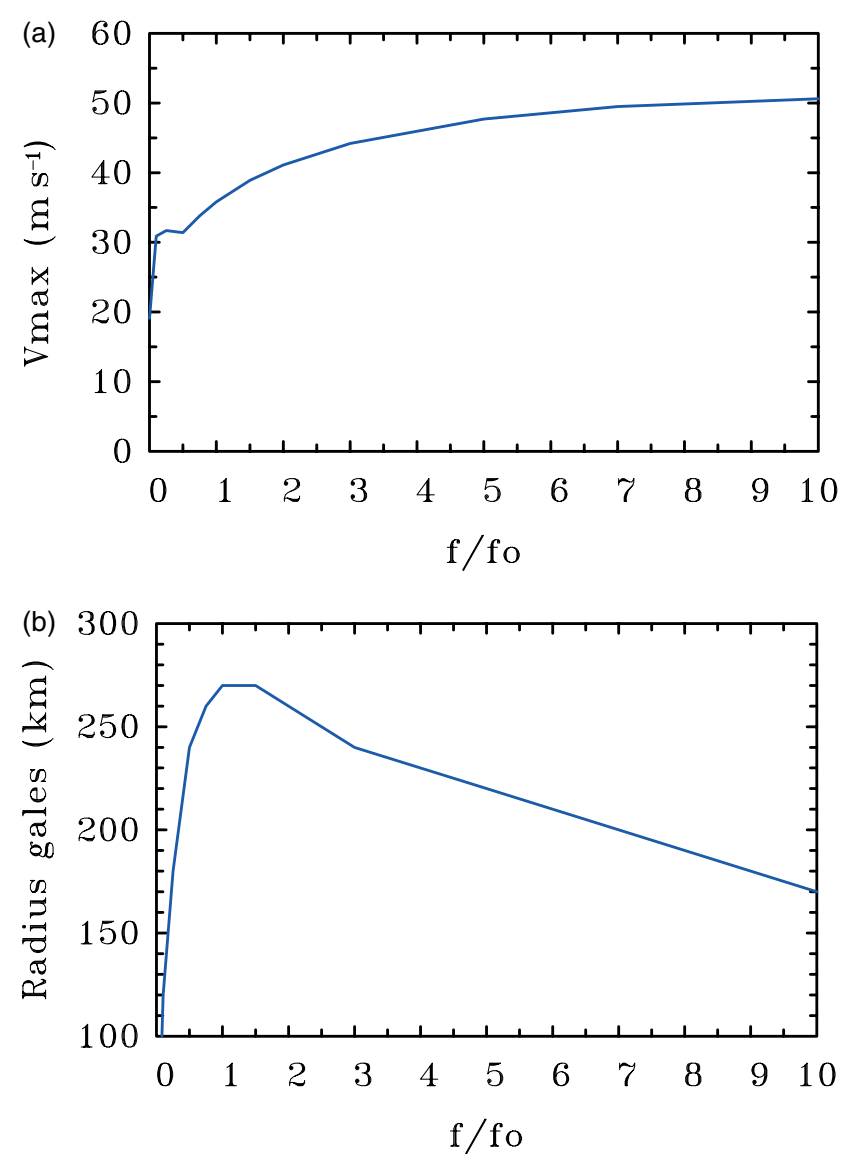

Figure 10. Variation with the background rotation of (a) the maximum tangential wind and (b) the radius of gale-force winds in the middle layer at $8 \mathrm{~d}$ in the set of calculations with fixed forcing. This figure is available in colour online at wileyonlinelibrary.com/journal/qj

vortex will weaken. Thus the radius of gales will contract with time.

As the background rotation rate is increased, there will be an increasing reservoir of planetary angular momentum for the vortex to draw upon, allowing it to sustain itself. However, inertial stability considerations dictate that, at least for a fixed forcing strength, the stronger the ambient rotation in the middle layer, the harder it is for air parcels in the outer region to be drawn inwards (subsection 1.2). Certainly, smaller radial displacements will lead to smaller increases in the maximum amplification of the angular velocity, but not necessarily to a smaller increase in the tangential wind speed. The reason is that the degree of amplification of the tangential wind speed depends both on the magnitude of the inward parcel displacement and the background rotation rate $^{* *}$. Thus smaller inward displacements do not necessarily lead to smaller-sized storms.

\section{Dependence on $f$ with fixed forcing}

It is evident from a comparison of Figures 3 and 7 that there is not a simple one-to-one correspondence between

**If a ring of air initially at radius $R$ in uniformly rotating fluid with angular velocity $f / 2$ is displaced to radius $r$ while conserving its absolute angular momentum, $r v+0.5 f r^{2}$, the tangential velocity at the new radius will be $v=(1 / 2 r) f\left(R^{2}-r^{2}\right)$, which depends on $f$. However, the relative amplification of the angular velocity is $v /\left(\frac{1}{2} r f\right)=R^{2} / r^{2}-1$, which is independent of $f$. 


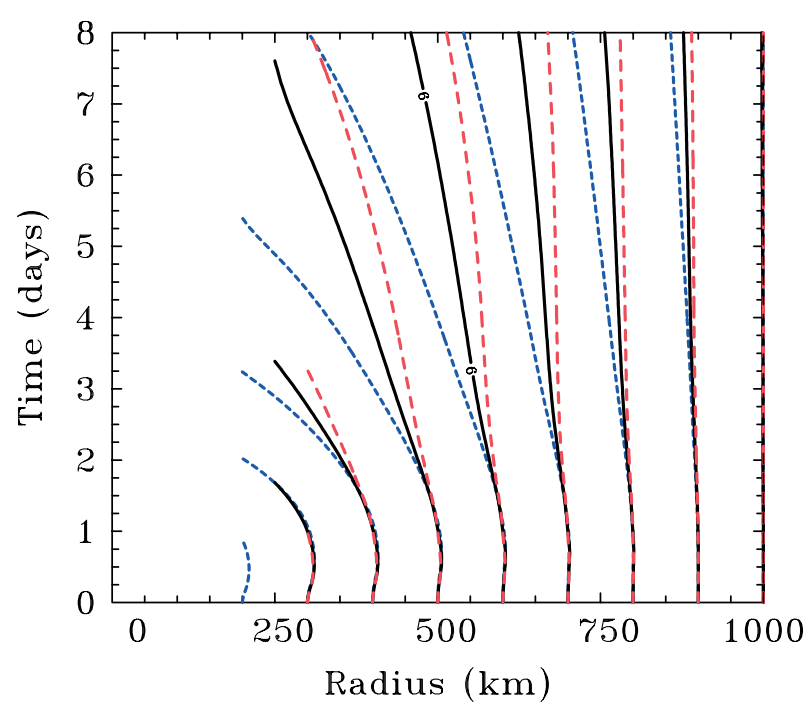

Figure 11. Time-radius plots of the trajectories in the middle layer in the calculations with $f=0.5 f_{0}$ (short dashed lines), $f=f_{0}$ (solid lines), and $f=1.5 f_{0}$ (long dashed lines). This figure is available in colour online at wileyonlinelibrary.com/journal/qj

the intensity and size of the vortex at a particular latitude and time and the 'effective forcing' at that time. For this reason, we examine now a set of simplified experiments with a modified version of the minimal axisymmetric model in which the radial and vertical structure of the diabatic heating rate are specified. By design, the effects of moisture are not considered explicitly. A heating distribution is prescribed in all the three layers, the specification of which is guided by the radial and vertical structure of the diagnosed heating rates in the mature stage in the foregoing moist calculation with $f=f_{0}$. The formula for the diabatic heating rate is given in Appendix A.3. The heating rate, which is the same in all experiments here, is multiplied by a simple function of time that allows the heating to build up exponentially from zero to a constant level on a time-scale of $\sim 24 \mathrm{~h}$. This procedure minimizes the spurious generation of inertia-gravity waves.

The experiments start from the initial vortex profile in Figure 2 and are distinguished by their latitude. The corresponding values of $f$ are: $f=0,0.1 f_{0}, 0.25 f_{0}, 0.5 f_{0}$, $0.75 f_{0}, 1.0 f_{0}, 1.5 f_{0}, 2.0 f_{0}, 3.0 f_{0}, 5.0 f_{0}, 7.0 f_{0}$, and $10.0 f_{0}$. The larger values of $f$ are clearly unrealistic for the Earth, but are used to provide an adequate survey of the vortex behaviour.

\subsection{Results}

Figure 9 shows time series of $v_{\max }$ in the middle layer for this set of calculations. It shows also time series of $r_{\text {gales }}$ in the middle layer. Again we see a clear dependence of the evolution of these quantities on $f$. Except in the calculations for $f \leq 0.25 f_{0}$, the intensity becomes approximately steady after about 4 days. In the case of zero background rotation, $f=0$, the intensity reaches a maximum after about 2.5 days and then steadily declines as the initial absolute angular momentum is progressively removed by surface friction.

Figure 10 shows $v_{\max }$ and $r_{\text {gales }}$ after eight days of integration as a function of $f$. The maximum tangential wind speed increases with increasing $f$. However, unlike the moist case described in section 4, there is no optimum background rotation rate for intensity, neither within the range of realistic values of $f$, nor beyond. This finding implies that the dependence of the effective forcing strength on the rotation rate found in the moist calculations is an important additional consideration for tropical cyclones.

Unlike intensity, there is an optimum background rotation rate for maximum vortex size (Figure 10(b)). For $f<0.75 f_{0}$ (i.e. to about $15^{\circ} \mathrm{N}$ ), $r_{\text {gales }}$ in the middle layer increases strongly with $f$. For $f$ between $f_{0}$ and $1.5 f_{0}$ (about $30^{\circ} \mathrm{N}$ ), its rate of increase slows and its value levels out at $270 \mathrm{~km}$. For larger values of $f, r_{\text {gales }}$ decreases.

Figure 11 shows time-radius plots of a passive tracer in the middle layer in the calculations with $f=0.5 f_{0}, f=f_{0}$ and $f=1.5 f_{0}$. As in Figure 5, the patterns of tracer movement are consistent with the idea discussed in section 1 that the inward radial displacements of air columns should decrease as the rotational constraint increases. In this case, the forcing strength does not change between these calculations. Moreover, the trajectories do not cross as they do in Figure 5, where the forcing strength does change.

\subsection{Interpretation}

An interpretation of the foregoing results is provided by an examination of the steady-state radial profiles of various quantities after 8 days of integration for different values of $f$. These quantities include: the radial, tangential and vertical velocity components; the absolute angular momentum; the tangential wind tendency due to the radial flux of absolute vorticity; and the agradient force ${ }^{\dagger \dagger}$. The profiles are shown in Figures 12-14 for a subset of the values for $f$ in Figure 9.

\subsubsection{Constraints on the secondary circulation}

The imposed forcing function leads to inflow in the middle layer beyond a certain radius $\left(130 \mathrm{~km}\right.$ for $f=0.25 f_{0}, 180 \mathrm{~km}$ for $f=0.5 f_{0}, 220 \mathrm{~km}$ for $f=f_{0}$, then decreasing to $130 \mathrm{~km}$ as $f$ increases; Figure 12(b)). The maximum inflow decreases in strength as $f$ increases to $f_{0}$ and the radius of the maximum increases. As $f$ increases beyond $f_{0}$, the inflow increases again in magnitude and the radius of the maximum inflow decreases. It is plausible that the initial decrease in strength and increase in the radius of maximum inflow are associated with the increase in the inertial stability, which makes it harder for the fixed forcing to draw air parcels inwards. However, as $f$ increases further, the increasing stability to radial motion confines the subsidence closer and closer to the forcing region (Figures $12(\mathrm{e}, \mathrm{f})$ ) so that, to the extent that the mass flux carried by the secondary circulation is not appreciably reduced in strength, the return radial inflow must increase in strength and its maximum must move inwards. Figures $12(\mathrm{e}, \mathrm{f})$ show that the maximum vertical velocity at the model interface levels, and hence the mass flux of the secondary circulation, is actually reduced in strength as $f$ increases, but clearly not sufficiently to reverse the increase in maximum radial inflow discussed above. As $f$ increases beyond $f_{0}$, the geometric effect takes over, i.e. the subsidence occurs over an area that decreases in proportion to the radius squared whereas the inflow increases inversely with radius. As a result, the maximum subsidence increases and the radius of the maximum inflow decreases.

\footnotetext{
${ }^{\dagger}$ The complete radial and tangential momentum equations are given in Nguyen et al. (2002). The absolute vorticity is equal to the radial gradient of absolute angular momentum divided by radius. The agradient force is the sum of radial pressure gradient force, and the centrifugal and Coriolis forces per unit mass.
} 

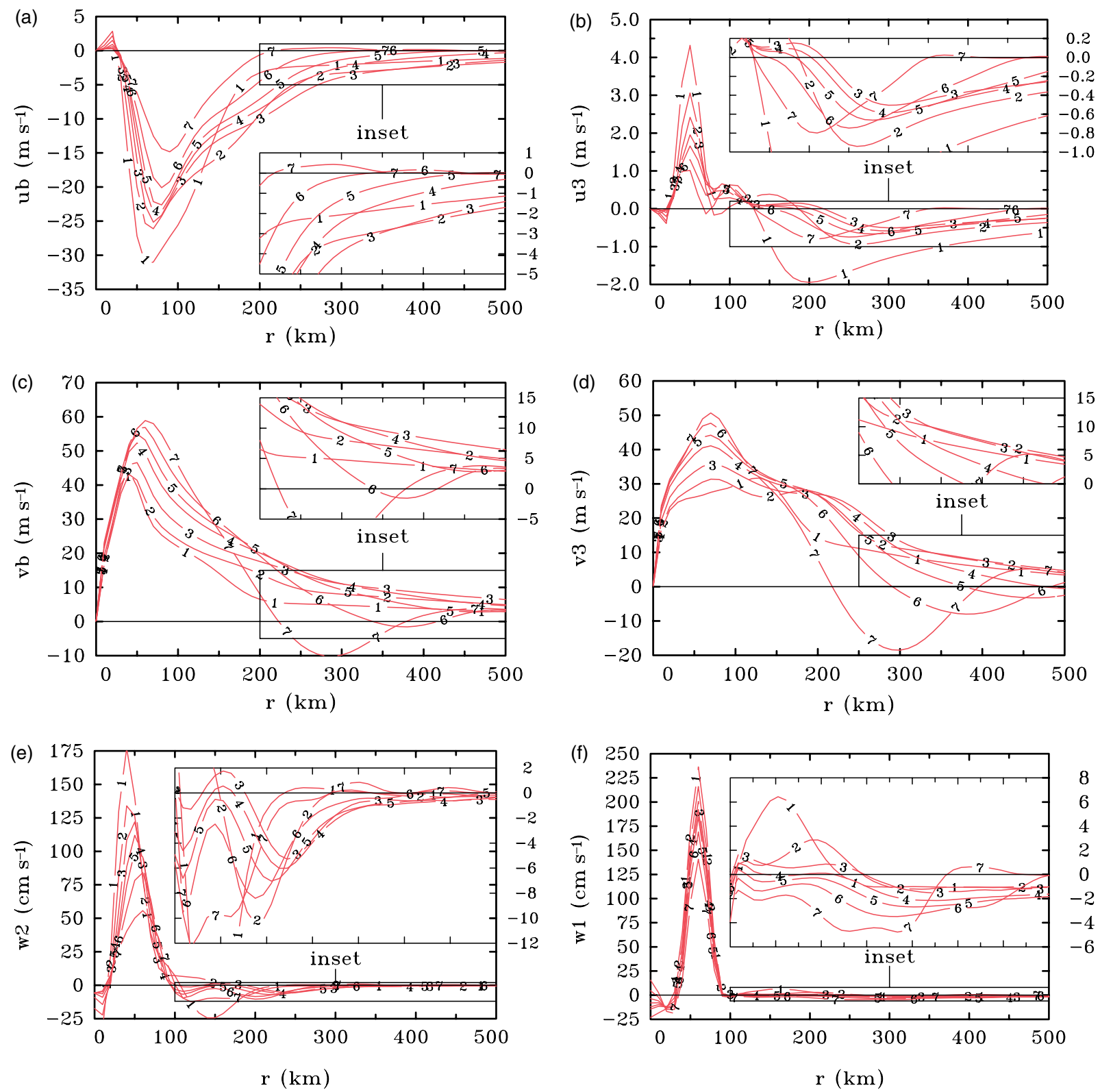

Figure 12. Steady-state radial profiles of radial wind speed in (a) the boundary layer and (b) the middle layer for selected values of $f$ after 8 days. (c) and (d) show the corresponding profiles for tangential wind speed, and (e) and ( $\mathrm{f}$ ) those of vertical velocity between the boundary layer and middle layer, and between the middle layer and upper layer, respectively. Curves labelled 1 to 7 respectively denote $f=0.25 f_{0}, 0.5 f_{0}, f_{0}, 2 f_{0}, 3 f_{0}, 5 f_{0}$, and $10 f_{0}$. The inset regions show an amplified view of the rectangular region outlined beyond a certain radius. This figure is available in colour online at wileyonlinelibrary.com/journal/qj

\subsubsection{Factors controlling size}

Figure 12(d) shows the tangential wind profiles after 8 days in the middle layer for selected values of $f$. As a preliminary to understanding the behaviour of these profiles as $f$ increases, we note that, beyond a certain radius, the spin-up of the tangential wind in the middle layer occurs solely because of inflow in regions where the radial gradient of absolute angular momentum, $M$, is positive, i.e. by the radial advection of $M$ divided by radius. The spin-up is moderated by the downward advection of $M$ by subsidence from the upper layer, where values of $M$ at any radius are typically less than in the middle layer because of prior frictional loss in the boundary layer. As $f$ increases, the maximum subsidence increases in strength because, as shown above, the secondary circulation becomes more confined radially. The effect of this subsidence on the tangential wind profile is most evident at background rotation rates exceeding $2 f_{0}$ (curves 5-7 in Figure 12(d)), where it leads to anticyclonic flow inside a radius of $500 \mathrm{~km}$.

At a given radius beyond about $150 \mathrm{~km}$, the tangential wind speed increases with increasing $f$ up to a certain value ( $f \leq f_{0}$ for $r<400 \mathrm{~km}, f \leq 0.5 f_{0}$ for $\left.r>400 \mathrm{~km}\right)$ and then decreases as $f$ increases further. The increase has to be associated with the radial advection of $M,-u(\partial M / \partial r)$, by the inflow in regions where $\partial M / \partial r>0$. Here $u$ is the radial component of flow and $r$ is the radius. While at most values of $r, \partial M / \partial r$ is mostly positive and increases with

Figure 13(b) shows that there is a small range of radii in which $\partial M / \partial r<0$. This relatively weak negative gradient arises because of the downward advection of $M$ from the upper layer, where values of $M$ are typically smaller than those in the middle layer. In these regions, of course, the local tendency of the tangential wind, $\partial v / \partial t$, is negative. 

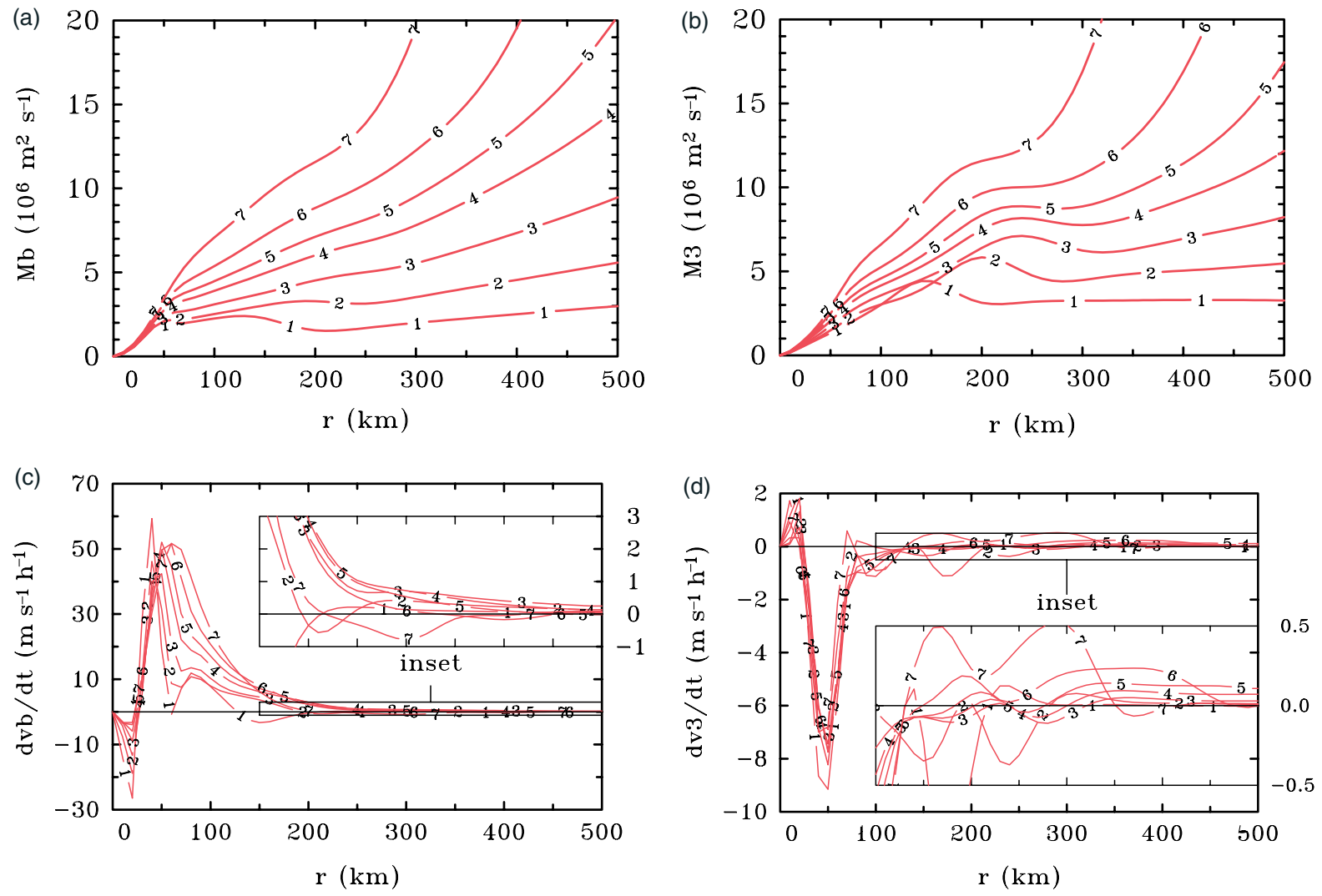

Figure 13. Steady-state radial profiles of the absolute angular momentum in (a) the boundary layer and (b) the middle layer after $8 \mathrm{~d}$ for the values of $f$ in Figure 12. (c) and (d) show the corresponding profiles of the time tendency of the tangential wind associated with the radial advection of absolute angular momentum. The inset region in each panel shows an amplified view of the rectangular region outlined beyond a certain radius. This figure is available in colour online at wileyonlinelibrary.com/journal/qj

$f$, the behaviour of $u$ as $f$ increases is more complicated (Figure 12(b)) and it is not possible to simply infer the change in $-u(\partial M / \partial r)$ as $f$ increases: one must do the calculation. For this reason, we show in Figure 13(d) the radial profiles of the tangential wind tendency associated with the radial advection of absolute angular momentum, $-(u / r)(\partial M / \partial r)$, in the middle layer for various values of $f$. It turns out that the variation of $-(u / r)(\partial M / \partial r)$ with $f$ is a strong function of radius, which is complicated by the fact that the range of radii for which $\partial M / \partial r<0$ varies with $f$ also. For example, in the radial range from 360 to $400 \mathrm{~km}$, $-(u / r)(\partial M / \partial r)$ increases with $f$ for values less than $5 f_{0}$ and then decreases, but at other radii the behaviour is different.

For small values of $f$, the radial advection of $M$ accounts for the largest contribution to the increase in the tangential wind speed with increasing $f$ outside the core region. For these values the subsidence is relatively weak in this region (Figure $12(\mathrm{~d})$ ) and the reduction of $M$ by the vertical advection of lower values of $M$ from the upper layer is correspondingly weak. However, as $f$ increases, this reduction of $M$ in the middle layer by downward advection becomes increasingly important. It is primarily these two competing effects that provide an explanation for the existence of an optimum size as shown in Figure 12(d).

\subsubsection{The role of the boundary layer}

In the boundary layer, the agradient force per unit mass, $F_{\text {agrad }}$, is negative beyond a radius of $70 \mathrm{~km}$ and has a minimum value just inside a radius of $100 \mathrm{~km}$, except for $f=0.25 f_{0}$ where this minimum occurs at a radius of about
$120 \mathrm{~km}$ (Figure 14). The minimum force decreases in value at first as $f$ increases, but it increases again as $f$ increases beyond $0.5 f_{0}$. In contrast, changes in the breadth of the agradient force profile as $f$ increases are complicated and depend on the range of radii one considers. A cursory examination of Figure 14, for example, suggests that the broadest profile is that for $f=0.25 f_{0}$, but a closer inspection of the inset region would suggest that the profile for $f=0.5 f_{0}$ is the broadest. What matters most is the maximum inward displacement of $M$ surfaces, taking account of the loss of $M$ en route (Smith et al., 2009). This displacement is determined by an inward radial integral of the sum of the agradient force and the frictional force, while the loss of $M$ is determined by a radial integral of the tangential momentum equation (e.g. Smith and Vogl, 2008). According to boundary-layer theory, the radial pressure gradient in the boundary layer is equal to that in the middle layer, which is directed inwards. The centrifugal, Coriolis and the radial frictional forces, which for cyclonic flow are all directed outwards, depend on the tangential wind speed in the boundary layer. Thus the radial and tangential velocity components in the boundary layer are determined by solving these two coupled equations that, in turn, depend on the radial pressure gradient in the middle layer.

\subsubsection{Factors controlling intensity}

Inside a radius of about $70 \mathrm{~km}$, the agradient force becomes positive (i.e. the tangential flow becomes supergradient) and the boundary-layer inflow rapidly decelerates and turns upwards to enter the middle layer. It is the vertical advection 


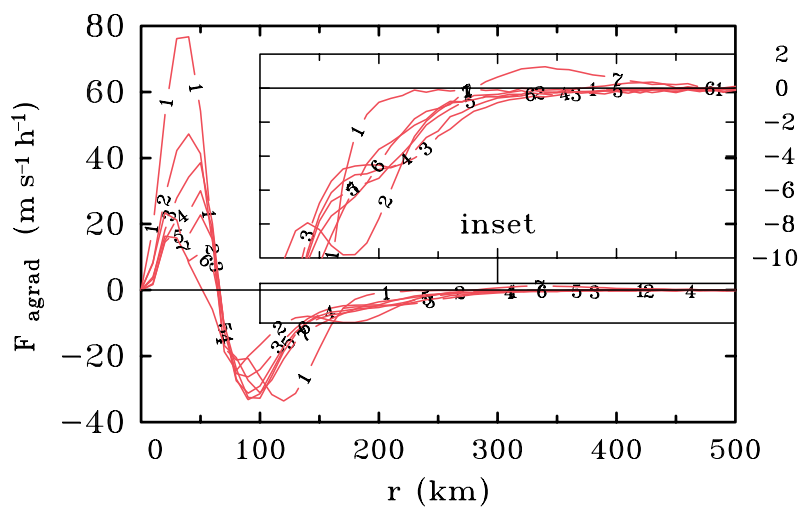

Figure 14. Steady-state radial profiles of the agradient force in the boundary layer after $8 \mathrm{~d}$ in the calculations with fixed forcing for the same values of $f$ as in Figure 12. The inset region shows an amplified view of the rectangular region outlined beyond a radius of $100 \mathrm{~km}$. This figure is available in colour online at wileyonlinelibrary.com/journal/qj

of absolute angular momentum out of the boundary layer that determines the maximum tangential wind speed in the middle layer as described for the moist case in section 5 .

It is evident from the foregoing discussion that the boundary layer and interior flow are tightly coupled and, for this reason, attempts to construct simple cause and effect arguments to explain the behaviour of the vortex as $f$ varies are fraught with danger! However, we attempt to describe the nature of the coupling that allows us to understand the behaviour of the fields in Figure 12 as $f$ varies.

In the boundary layer, the maximum radial inflow decreases and the radius at which it occurs increases as $f$ increases (Figure 12(a)). In addition, its radial profile broadens with increasing $f$ for values less than $f_{0}$ and decreases for larger values of $f$. This behaviour influences the radial profile of $-(u / r)(\partial M / \partial r)$ and thereby the profile of tangential wind. There are two main opposing effects that determine the radial profile of $v$ : one is the radial advection of absolute angular momentum and the other is the surface friction. Figure 13(a) shows that, as $f$ increases, so does $\partial M / \partial r$, but the maximum radial velocity decreases (Figure 12(a)). Moreover, the loss of $M$ due to surface friction increases as $M$ itself increases, but it decreases as the radial wind speed increases. The last result is essentially because, with higher inflow, air parcel trajectories undergo fewer revolutions per unit radial displacement and are therefore shorter in length, so that friction has less distance over which to slow the parcels down. Our calculations show that, as $f$ increases, the larger gradient of $M$ outweighs the reduced radial inflow and larger loss of $M$ to the surface, so that the largest tangential wind in the boundary layer occurs for the largest values of $f$. Thus, the fact that the spin-up of the tangential wind speed at inner radii in the middle layer occurs by vertical advection of $M$ (or tangential momentum) from the boundary layer explains why the maximum tangential wind speed in the middle layer (Figure 9(a)) increases as $f$ increases.

\section{Discussion}

While the TLM ideas have provided a useful starting point for investigating the dependence on the latitude of intensity and size of tropical cyclones, a simple interpretation for the dependence found in our model calculations does not seem possible. The reason is rooted in the tight coupling that exists between the flow in the middle layer and that in the boundary layer. Nevertheless, in the simplest problem with fixed forcing, we have been able to articulate the key components of the coupling that help to understand the results. In the moist problem discussed in section 4 , there is an additional tier of complexity that results from the additional coupling between the forcing associated with the diabatic heating and the wind distribution in the boundary layer. The link between the heating and the winds arises from the dependence of the surface moisture flux on both the near-surface wind speed and the moisture disequilibrium between the specific humidity of near-surface air and the saturation specific humidity at the sea-surface temperature and pressure.

\section{Conclusions}

This article has sought to develop a basic framework for understanding the factors that influence the intensity and size of tropical cyclones, as characterized by the maximum tangential wind speed and the radius of gales, respectively. For simplicity we have focused on the axisymmetric aspects of the dynamics.

The classical TLM ideas provide a helpful starting point for these investigations. These ideas are based on the premise that there are two fundamental requirements for vortex amplification: a source of rotation and some forcing mechanism to generate an overturning circulation to amplify the rotation. When switched on, the forcing mechanism acts to draw fluid parcels inwards, thereby increasing the tangential wind speed by conservation of absolute angular momentum. The increasing centrifugal and Coriolis forces associated with the increasing tangential velocity component tend to resist these displacements. The ultimate degree of amplification of the angular velocity during this adjustment period depends on how far rings of fluid can be drawn inwards and on the background rotation rate. How far rings of fluid can be drawn inwards depends, inter alia, on the forcing strength. If the forcing is sufficiently large for a given rotation rate, rings of fluid may be drawn in to relatively small radii before the centrifugal and Coriolis forces balance the radial pressure gradient induced by the forcing. If the forcing is comparatively weak, or if the rotation rate is sufficiently strong, this balance may be achieved before the radial displacement is very large, so that a significant amplification of the background rotation will not be achieved. Of course, if there is no background rotation, there will be no amplification, and if the background rotation is very weak, the centrifugal and/or Coriolis forces may never become large enough to balance the radial pressure gradient.

Tropical cyclones have a large quasi-balanced component to their evolution and the forcing mechanism, rather than being impulsive, is associated with the radial gradient of the diabatic heating rate that results from the collective effects of deep vortical convective clouds. In this situation, the resistance to radial motion is associated more with the inertial stability of the vortex plus the ambient rotation.

Using a suite of idealized calculations with a minimal axisymmetric tropical-cyclone model, we have shown that there is an optimum ambient rotation, characterized by the Coriolis parameter, for the largest vortex intensity and for the largest vortex size. The former finding is consistent with earlier results of DeMaria and Pickle, but the latter is not. At first sight these findings appear to be broadly 
consistent with expectations from the TLM ideas. However, we have identified a number of reasons why these ideas are of limited applicability to tropical cyclones. For one thing, the interpretations in the moist calculations described herein are complicated by the fact that the effective forcing is found to change with latitude, making it difficult to anticipate the outcome in a simple way.

A more serious limitation of the TLM ideas is the fact that the spin-up of the maximum tangential winds in the tropical-cyclone inner core occurs in the boundary layer, where, because of friction, there is a net inward force, irrespective of the perceived inertial resistance to radial motion. In this layer, absolute angular momentum is not conserved so that the TLM ideas do not strictly apply. In consequence of this spin-up mechanism, a simple interpretation for the dependence of intensity and size of tropical cyclones remains to us elusive. However, based on our findings, we have offered an articulation of the key components of the dynamics which help to interpret the foregoing dependence. Because of several competing effects, one has to do the relevant calculation to determine which effects outweigh the others for a particular level of ambient rotation.

When the forcing is held fixed, we found that the maximum intensity increases monotonically with increasing rotation rate, even for unrealistically large rotation rates. However, there is an optimum rotation rate to produce the vortex with the largest size. We were able to provide an understanding of this behaviour, based on diagnostics of the competing processes involved.

We found that, in calculations starting with the same modest-sized initial vortex, there is not a one-to-one correspondence between the vortex intensity and size during the entire period of development. This lack of a relationship is in line with the observational study of Weatherford and Gray. However, we did find that for the more intense storms $\left(v_{\max }>35 \mathrm{~m} \mathrm{~s}^{-1}\right)$, there is an approximate linear increase in the radius of gales with intensity. The occurrence of intense midget storms leads us to be cautious about the generality of this relationship.

The foregoing results point to the existence of dynamical constraints that are not explicitly included in existing theories for the maximum possible intensity of hurricanes. This study is a first step to try to understand hitherto unexplored aspects of the rotational constraints on tropicalcyclone intensification and maturity. We purposefully began with a very simple model in an attempt to isolate fundamental processes. The calculations described have been repeated using a more sophisticated three-dimensional, non-hydrostatic, mesoscale model and the findings are broadly consistent with those obtained herein. The results of these calculations will be submitted for publication in due course.

\section{Acknowledgements}

We thank two anonymous reviewers for their perceptive and constructive comments on the original version of this manuscript. RKS and CWS acknowledge financial support for hurricane research from the German Research Council (Deutsche Forschungsgemeinschaft). MTM acknowledges the support of grant no. N00014-03-1-0185 from the US Office of Naval Research, NOAA's Hurricane Research
Division, and the US National Science Foundation grants ATM-0649944 and ATM-0715426.

\section{Appendix}

\section{A.1. Trajectory calculations}

In order to show trajectories of air parcels, the advection equation (Eq. A.1) was solved for the passive tracer, $X$, with its initial value equal to the initial radius in each layer:

$$
\frac{\partial X}{\partial t}=-u \frac{\partial X}{\partial r}
$$

This equation was solved using the Adams-Bashforth thirdorder scheme, with a time step of $6 \mathrm{~s}$ as for all other prognostic variables (Zhu et al., 2001).

\section{A.2. Diabatic heating rate calculation}

The maximum magnitude of the negative radial gradient of the diabatic heating rate, i.e. $\max (-\partial \dot{\theta} / \partial r)$, has been chosen as an appropriate measure for the effecive forcing. The reasons for it are explained in section 6 . The thermodynamic equation is solved for $\theta$ without the diabatic term $Q_{\theta}$.

$$
\frac{\partial \theta}{\partial t}=-u \frac{\partial \theta}{\partial r}-\dot{\sigma} \frac{\partial \theta}{\partial \sigma}+Q_{\theta}
$$

After each time integration step, heating contributions from the cumulus parametrization scheme and explicit condensation on the grid-scale are added to $\theta$. The diabatic heating rate for each time step is then the difference of both values of $\theta$ divided by the integration time step, which was taken to be $6 \mathrm{~s}$ for all calculations. From this value, the maximum negative radial gradient can easily be calculated.

\section{A.3. Fixed forcing specification}

In the calculations with a fixed forcing, the diabatic heating rate was prescribed by the formula

$$
\dot{\theta}=2 a Q_{0}\left(\frac{R-0.5 R_{0}}{R_{0}}\right)^{4} \exp \left\{-\left(\frac{R-0.5 R_{0}}{R_{0}}\right)^{4}\right\} T(t),
$$

where $T(t)=1-\exp \left\{-\left(t / t_{0}\right)^{2}\right\}, \quad t$ is the time, $Q_{0}=$ $600 \mathrm{~K} \mathrm{day}^{-1}, R_{0}=40 \mathrm{~km}, t_{0}=24 \mathrm{~h}$, and $a$ is a factor specific for each model level. This diabatic term was added in the middle $(a=1.1)$ and upper layer $(a=1.8)$. For the boundary layer $(a=3.3)$, a slightly different formula for the diabatic heating rate was used, which takes into account that its maximum is closer to the storm centre:

$$
\dot{\theta}=2 a Q_{0}\left(\frac{R}{R_{0}}\right)^{4} \exp \left\{-\left(\frac{R}{R_{0}}\right)^{4}\right\} T(t) .
$$

The spatial variation of $\dot{\theta}$ is similar to the one used by Möller and Smith (1994). The time dependence, $T(t)$, is included to avoid the shock of imposing a finite value of diabatic heating at the initial instant. 


\section{References}

Arakawa A. 1969. 'Parameterization of cumulus convection'. In Proc. WMO/IUGG Sympos. Numerical Weather Prediction, Tokyo, 26 November-4 December 1968. Japan Meteorol. Agency IV 8: 1-6.

Bister M, Emanuel KA. 1998. Dissipative heating and hurricane intensity. Meteorol. Atmos. Phys. 65: 233-240.

Bui HH, Smith RK, Montgomery MT, Peng J. 2009. Balanced and unbalanced aspects of tropical-cyclone intensification. Q. J. R. Meteorol. Soc. 135: 1715-1731.

Camp JP, Montgomery MT. 2001. Hurricane maximum intensity: Past and present. Mon. Weather Rev. 129: 1704-1717.

Charney JG. 1973. Planetary fluid dynamics. In Dynamic Meteorology, Morel P. (ed.) Reidel: Dordrecht, Netherlands.

DeMaria M, Pickle JD. 1988. A simplified system of equations for simulation of tropical cyclones. J. Atmos. Sci. 45: 1542-1554.

Emanuel KA. 1986. An air-sea interaction theory for tropical cyclones. Part I: Steady state maintenance. J. Atmos. Sci. 43: 585-604.

Emanuel KA. 1988. The maximum intensity of hurricanes. J. Atmos. Sci. 45: $1143-1155$.

Emanuel KA. 1989. The finite amplitude nature of tropical cyclogenesis. J. Atmos. Sci. 46: 3431-3456.

Emanuel KA. 1995. Sensitivity of tropical cyclones to surface exchange coefficients and a revised steady-state model incorporating eye dynamics. J. Atmos. Sci. 52: 3969-3976.

Emanuel KA. 1997. Some aspects of hurricane inner-core dynamics and energetics. J. Atmos. Sci. 54: 1014-1026.

Emanuel KA. 2004. Tropical cyclone energetics and structure. In Atmospheric Turbulence and Mesoscale Meteorology, Fedorovich E, Rotunno R, Stevens B. (eds.) Cambridge University Press: Cambridge, UK.

Frisius T. 2006. Surface-flux-induced tropical cyclogenesis within an axisymmetric atmospheric balanced model. Q. J. R. Meteorol. Soc 132: $2603-2623$.

Holland GH. 1997. The maximum potential intensity of tropical cyclones. J. Atmos. Sci. 54: 2519-2541.

Merrill RT. 1984. A comparison of large and small tropical cyclones. Mon. Weather Rev. 112: 1408-1418.

Möller JD, Smith RK. 1994. The development of potential vorticity in a hurricane-like vortex. Q. J. R. Meteorol. Soc. 120: 1255-1265.

Montgomery MT, Smith RK. 2011. Paradigms for tropical-cyclone intensification. Q. J. R. Meteorol. Soc. submitted.

Montgomery MT, Nicholls ME, Cram TA, Saunders AB. 2006. A vortica hot tower route to tropical cyclogenesis. J. Atmos. Sci. 63: 355-386.

Montgomery MT, Nguyen SV, Smith RK. 2009. Do tropical cyclones intensify by WISHE? Q. J. R. Meteorol. Soc. 135: 1697-1714.

Morton BR. 1966. Geophysical vortices. Prog. Aeronaut. Sci. 7: 145-194.

Nguyen CM, Smith RK, Zhu H, Ulrich W. 2002. A minimal axisymmetric hurricane model. Q. J. R. Meteorol. Soc. 128: 2641-2661.

Nguyen SV, Smith RK, Montgomery MT. 2008. Tropical-cyclone intensification and predictability in three dimensions. Q. J. R. Meteorol. Soc. 134: 563-582.

Ooyama KV. 1969. Numerical simulation of the life cycle of tropical cyclones. J. Atmos. Sci. 26: 3-40.

Ooyama KV. 1982. Conceptual evolution of the theory and modeling of the tropical cyclone. J. Meteorol. Soc. Japan 60: 369-379.
Persing J, Montgomery MT. 2003. Hurricane superintensity. J. Atmos. Sci. 60: 2349-2371.

Rayleigh Lord (William Thomson). 1917. On the dynamics of revolving fluids. Proc. R. Soc. London 93: 148-154.

Rotunno R, Emanuel KA. 1987. An air-sea interaction theory for tropical cyclones. Part II: Evolutionary study using a non-hydrostatic axisymmetric numerical model. J. Atmos. Sci. 44: 542-561.

Sanger NT. 2011. 'An observational study of tropical-cyclone spin-up in supertyphoon Jangmai and hurricane Georges'. PhD thesis. Naval Postgraduate School: Monterey, California, USA.

Schubert WH, Hack JJ. 1983. Transformed Eliassen balanced vortex model. J. Atmos. Sci. 40: 1571-1583.

Shapiro LJ, Willoughby H. 1982. The response of balanced hurricanes to local sources of heat and momentum. J. Atmos. Sci. 39: 378-394.

Shin S, Smith RK. 2008. Tropical-cyclone intensification and predictability in a minimal three-dimensional model. Q. J. R. Meteorol. Soc. 134: $1661-1671$.

Smith RK, Leslie LM. 1976. Thermally driven vortices: A numerical study with application to dust devils. Q. J. R. Meteorol. Soc. 102: 791-804.

Smith RK, Leslie LM. 1978. Tornadogenesis. Q. J. R. Meteorol. Soc. 104: 189-198.

Smith RK, Montgomery MT. 2008. Balanced boundary layers in hurricane models. Q. J. R. Meteorol. Soc. 134: 1385-1395.

Smith RK, Montgomery MT. 2010. Hurricane boundary-layer theory. Q. J. R. Meteorol. Soc. 136: 1665-1670.

Smith RK, Vogl S. 2008. A simple model of the hurricane boundary layer revisited. Q. J. R. Meteorol. Soc. 134: 337-351.

Smith RK, Montgomery MT, Vogl S. 2008. A critique of Emanuel's hurricane model and potential intensity theory. Q. J. R. Meteorol. Soc. 134: $551-561$.

Smith RK, Montgomery MT, Nguyen SV. 2009. Tropical cyclone spin-up revisited. Q. J. R. Meteorol. Soc. 135: 1321-1335.

Turner JS. 1966. The constraints imposed on tornado-like vortices by the top and bottom boundary conditions. J. Fluid. Mech. 25: $377-400$.

Turner JS, Lilly DK. 1963. The carbonated-water tornado vortex. J. Atmos. Sci. 20: 468-471.

Vogl S, Smith RK. 2009. Limitations of a linear model for the hurricane boundary layer. Q. J. R. Meteorol. Soc. 135: 839-850.

Weatherford CL, Gray WM. 1988. Typhoon structure as revealed by aircraft reconnaissance. Part I. Data analysis and climatology. Mon. Weather Rev. 116: 1032-1043.

Willoughby HE. 1979. Forced secondary circulations in hurricanes. J. Geophys. Res. 84: 3173-3183.

Willoughby HE. 1990. Gradient balance in tropical cyclones. J. Atmos. Sci. 47: 465-489.

Wirth V, Dunkerton TJ. 2006. A unified perspective on the dynamics of hurricanes and monsoons. J. Atmos. Sci. 63: 2529-2547.

Yamasaki M. 1968. Numerical simulation of tropical cyclone development with the use of the primitive equations. J. Meteorol. Soc. Japan 46: 202-214.

Zhang D-L, Liu Y, Yau MK. 2001. A multi-scale numerical study of hurricane Andrew (1992). Part IV: Unbalanced flows. Mon. Weather Rev. 129: $92-107$

Zhu H, Smith RK, Ulrich W. 2001. A minimal three-dimensional tropical cyclone model. J. Atmos. Sci. 58: 1924-1944. 